\title{
Reducing Modal Consequence Relations
}

\author{
Marcus Kracht \\ February 3, 2008
}

\begin{abstract}
In this paper we will investigate the possibility of reducing derivability in a modal consequence relation to consistency in unimodal and polymodal $\mathbf{K}$ by means of so-called reduction functions. We will present new and easy methods to prove standard results on decidability, complexity, finite model property, interpolation and Halldén-completeness using only the reduction functions. Some new results on complexity of modal logics will be established. All proofs are in addition constructive.
\end{abstract}

\section{Introduction}

Proofs of the finite model property in modal logic typically proceed via the detour of the canonical models or the weak canonical models, where the weak canonical models are based on finitely many sentence letters only. The alternatives to this method, tableaux methods or normal forms, do not seem to be as flexible as the methods designed to produce finite models from the canonical model. Yet, canonical models too have disadvantages. They are highly abstract structures. Moreover, even when it is finite (for example when the logic determines a locally finite variety) it may be the case that the structure of a weak canonical model or the structure of a finitely generated free algebra cannot be determined if the logic is undecidable. So, this method of proof is highly inconstructive. This is not only a theoretical disadvantage. Typically, explicit solutions are easier to understand. Tableau systems can be used quite effectively for the standard systems. However, tableau calculi for many logics extending $\mathbf{K}$ are often rather ad-hoc. They use a mixture of closure rules (rules to be applied at a particular world) and step rules (rules to be applied when moving to a successor). 
The present paper presents a combinatorial method that uses only the reduction of provability in a stronger system into provability of a weaker system. Though the proof of the success of the method typically involves model theoretic arguments, it is nevertheless finitistic. Its advantages are manifold. It is constructive, explicit, and yields proofs of the finite model property, decidability, complexity and interpolation, to name a few. Once the reduction of one logic $M$ to a weaker $\operatorname{logic} L$ is shown, many properties of $L$ are shown to transfer to $M$ with no additional effort. The proof of interpolation, for example, is so simple that it reduces interpolation of the standard systems to a mere corollary of the method. (Compare this, for example, with the criterion developed in [14], which is difficult to state let alone apply.) This method is not new. It has been used, for example, by Balbiani and Herzig in [1] and De Giacomo in [5]. However, what is new is the systematic study of the method, and the observation that it can be used for much more than just the proof of finite model property.

I should say here that not everything presented here is new. In particular, most of the results of Sections 3, 5 and 6 have been anticipated in [7], though usually not in as general a form as they appear here. The results of Section 4 are, however, entirely new. Moreover, in an appendix we show how to give a completely constructive proof of a well-known theorem by Kit Fine on subframe logics. It is based on the ideas outlined here. Finally, I wish to thank Stefan Baier for his help with number theory and an anonymous referee for useful comments.

\section{Preliminaries}

We consider the language of modal logic with any number of unary modal operators. The vocabulary consists of a set of variables, $V:=\left\{p_{i}: i \in \omega\right\}$, and the functors $\perp, \neg, \wedge$ and $\square_{i}, i<\kappa$. Here $\kappa$ is a cardinal number. If $\kappa=1$ we will write $\square$ rather than $\square_{0}$. Formulae are built in the usual way; we use lower case Greek letters $(\varphi, \psi, \ldots)$ to denote formulae, and upper case Greek letters $(\Delta, \Gamma, \ldots)$ to denote sets of formulae. We use the standard notation $\Delta ; \varphi$ to denote $\Delta \cup\{\varphi\}$ and $\varphi ; \psi$ for $\{\varphi\} \cup\{\psi\}$. The following symbols, also frequently used, are treated as abbreviations:

$$
\begin{array}{ll}
\top & :=\neg \perp \\
\varphi \vee \psi & :=\neg(\neg \varphi \wedge \neg \psi) \\
\varphi \rightarrow \psi & :=\neg(\varphi \wedge \neg \psi) \\
\varphi \leftrightarrow \psi & :=(\varphi \rightarrow \psi) \wedge(\psi \rightarrow \varphi) \\
\diamond_{j} \varphi & :=\neg \square_{j} \neg \varphi
\end{array}
$$


$\operatorname{var}(\varphi)$ denotes the set of variables occurring in $\varphi$ and $s f(\varphi)$ the set of subformulae of $\varphi$. The notation $\operatorname{var}[\Delta]$ and $s f[\Delta]$ are self-explanatory. (We remark here that if $f$ is a function, and $X$ a subset of the domain of $f$ we write $f[X]:=\{f(x): x \in X\}$.) The modal depth, $d p(\varphi)$ is defined as follows.

$$
\begin{array}{ll}
d p\left(p_{i}\right) & :=0 \\
d p(\perp) & :=0 \\
d p(\neg \varphi) & :=d p(\varphi) \\
d p(\varphi \wedge \psi) & :=\max \{d p(\varphi), d p(\psi)\} \\
d p\left(\square_{j} \varphi\right) & :=1+d p(\varphi)
\end{array}
$$

The set of all formulae is denoted by $\mathcal{F}_{\kappa}$. A substitution is a function $\sigma: V \rightarrow \mathcal{F}_{\kappa}$. The effect of applying $\sigma$ to $\varphi(\Delta)$ is denoted by $\varphi^{\sigma}\left(\Delta^{\sigma}\right)$.

A consequence relation is a relation $\vdash \subseteq \wp\left(\mathfrak{F}_{\kappa}\right) \times \mathfrak{F}_{\kappa}$ such that

1. If $\varphi \in \Delta$ then $\Delta \vdash \varphi$.

2. If $\Delta \vdash \varphi$ and $\Delta \subseteq \Delta^{\prime}$ then $\Delta^{\prime} \vdash \varphi$.

3. If $\Delta \vdash \varphi$ for all $\varphi \in \Sigma$ and $\Sigma \vdash \psi$ then $\Delta \vdash \psi$.

$\vdash$ is structural if $\Delta \vdash \varphi$ implies $\Delta^{\sigma} \vdash \varphi^{\sigma}$ for every substitution $\sigma \cdot \vdash$ is finitary if $\Delta \vdash \varphi$ implies that there exists a finite $\Delta_{0} \subseteq \Delta$ such that $\Delta_{0} \vdash \varphi$. In sequel, all consequence relations are finitary and structural. A rule is a pair $\rho=\langle\Delta, \varphi\rangle$, where $\Delta \subseteq \mathfrak{F}_{\kappa}$ and $\varphi \in \mathfrak{F}_{\kappa} . \rho$ is finitary if $\Delta$ is finite. Examples of rules are MP := $\left\langle\left\{p_{0}, p_{0} \rightarrow p_{1}\right\}, p_{1}\right\rangle$ and $\mathrm{MN}_{j}:=\left\langle\left\{p_{0}\right\}, \square_{j} p_{0}\right\rangle$. Let $R$ be a set of finitary rules. We denote by $\vdash^{R}$ the smallest finitary structural consequence relation containing $R$. $r^{R}$ can be described as follows. Call an $R$-proof of $\varphi$ from $\Delta$ a finite sequence $\left\langle\delta_{i}: i<n\right\rangle$ such that

1. $\delta_{n-1}=\varphi$.

2. For all $i<n, \delta_{i} \in \Delta$ or there exists a subset $\Sigma \subseteq\left\{\delta_{j}: j<i\right\}$ such that $\left\langle\Sigma, \delta_{i}\right\rangle$ is a substitution instance of some element of $R$.

The following is stated without proof (see [7] for a complete proof).

Proposition $1 \Delta \vdash^{R} \varphi$ iff there exists an $R$-proof of $\varphi$ from $\Delta$.

We define a modal logic as a set of formulae. We assume that $A$ is a set of formulae which together with MP axiomatises classical propositional logic. 
Definition 2 A normal $\kappa-$ modal logic is a subset $L$ of $\mathfrak{F}_{\kappa}$ which contains $A$, the formulae $\square_{j}\left(p_{0} \rightarrow p_{1}\right) \rightarrow\left(\square_{j} p_{0} \rightarrow \square_{j} p_{1}\right)$, and is closed under substitution, and the rules $M P$ and $M N_{j}, j<\kappa$. The smallest $\kappa-$ normal modal logic is denoted by $\boldsymbol{K}_{\kappa}$.

We shall denote modal logics in sequel by upper case Roman letters, for example $L, M$ etc. Moreover, we will often speak of the rule MN, by which we denote the set of rules $\mathrm{MN}_{j}, j<\kappa$. Now let $L$ be a normal logic and $\Delta$ a set of formulae; the smallest normal logic containing $L$ and $\Delta$ is denoted by $L \oplus \Delta$. (With particular axioms such as $\mathbf{4}, \mathbf{T}$ etc. the notation $L . \Delta$ is also used.) The notions of frame and general frame are as usual. A general frame is a triple $\mathfrak{F}=\langle F, R, U\rangle$, where $F$ is a set, $R$ a function from $\kappa$ into subsets of $F^{2}$, and $U \subseteq \wp(F)$ closed under relative complement, intersection and the operations $\tau_{j}(A):=\{y:$ if $y R(j) x$ then $x \in A\}$, $j<\kappa$. We shall write $\triangleleft_{j}$ or $\triangleleft_{j}$ (with or without the index, when no confusion arises) to denote $R(j)$. If $U=\wp(F)$ we call $\mathfrak{F}$ a Kripke-frame and suppress mentioning $U$. A valuation into $\mathfrak{F}$ is a function $\beta: V \rightarrow U$. A triple $\mathfrak{M}=\langle\mathfrak{F}, \beta, x\rangle$ where $\mathfrak{F}$ is a frame, $\beta$ a valuation into $\mathfrak{F}$ and $x \in F$ is called a local model, the pair $\mathfrak{N}=\langle\mathfrak{F}, \beta\rangle$ a global model. In case, where $\mathfrak{M}=\langle\mathfrak{F}, \beta, x\rangle$ and $\mathfrak{N}=\langle\mathfrak{F}, \beta\rangle, \mathfrak{M}$ is a local expansion of $\mathfrak{N}$. Given a local model $\mathfrak{M}=\langle\mathfrak{F}, \beta, x\rangle$ and a formula $\varphi$, we define $\mathfrak{M}=\varphi$ as follows.

1. $\mathfrak{M} \not \models \perp$

2. $\mathfrak{M} \vDash \neg \varphi$ iff $\mathfrak{M} \not \models \varphi$.

3. $\mathfrak{M} \vDash \varphi \wedge \varphi^{\prime}$ iff $\mathfrak{M} \vDash \varphi$ and $\mathfrak{M} \vDash \varphi^{\prime}$.

4. $\mathfrak{M} \vDash \square_{j} \varphi$ iff for all $y$ such that $x R(j) y:\langle\mathfrak{F}, \beta, y\rangle \vDash \varphi$.

$\mathfrak{M}$ is a (local) $L$-model, if $\mathfrak{M} \vDash L$. We define $\mathfrak{N} \vDash \varphi$ iff $\mathfrak{M} \vDash \varphi$ for all local extensions $\mathfrak{M}$ of $\mathfrak{N}$.

Let $\vdash$ be a consequence relation. Then we put $\operatorname{Taut}(\vdash):=\{\varphi: \varnothing \vdash \varphi\}$. We call $\vdash$ a normal modal consequence relation if Taut $(\vdash)$ is a normal modal logic. We associate with a normal logic $L$ two special consequence relations, denoted by $\vdash_{L}$ and $\vdash_{L}$. The first is called the local consequence relation of $L$ and is defined as follows. $\Delta \vdash_{L} \varphi$ iff $\varphi$ is derivable from $\Delta \cup L$ by means of MP. $\vdash_{L}$ is called the global consequence relation of $L$ and $\Delta \Vdash_{L} \varphi$ iff $\varphi$ can be derived from $\Delta \cup L$ by means of MP and MN. (These consequence relations are not necessarily distinct. In fact, they are equal iff $p_{0} \rightarrow \square_{j} p_{0} \in L$ for all $j<\kappa$.) A (global) $L-$ proof of $\varphi$ from $\Delta$ is a proof of $\varphi$ from $\Delta \cup L$ using MP (MP and MN). 
The following is a consequence of the general completeness theorem of modal logic (with respect to general frames). We shall not prove it here, since it will not be used in sequel.

Proposition 3 Let L be a normal modal logic. Then the following holds.

1. $\Delta \vdash_{L} \varphi$ iff for all local $L-$ models $\mathfrak{M}:$ if $\mathfrak{M} \vDash \Delta$ then $\mathfrak{M} \vDash \varphi$.

2. $\Delta \Vdash_{L} \varphi$ iff for all global L-models $\mathfrak{N}$ : if $\mathfrak{N} \vDash \Delta$ then $\mathfrak{N} \vDash \varphi$.

We will also make use of the notion of a compound modality. A compound modality is a term in one variable, built up using only $\wedge$ and $\square_{j}$. Examples are $\square_{0} p_{0}, p_{0} \wedge \square_{1} p_{0}, \square_{1} \square_{0} p_{1}$ etc. Compound modalities behave like unary modal operators. We use $\boxplus$ as a variable over compound modalities. Now let $\sigma \in \kappa^{*}$ be a finite sequence of elements of $\kappa$. We define inductively the symbol $\square^{\sigma}$ as follows. (Here, $\varepsilon$ is the empty sequence.)

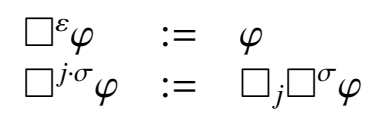

Finally, for a set $S \subseteq \kappa^{*}$ we put

$$
\square^{S} \varphi:=\left\{\square^{\sigma} \varphi: \sigma \in S\right\}
$$

Generally, we use the convention that a finite set of formulae $\Delta$ also denotes the conjunction $\wedge \Delta$. Hence, if $S$ is finite we also have

$$
\square^{S} \varphi=\bigwedge\left\langle\square^{\sigma} \varphi: \sigma \in S\right\rangle
$$

The following is easy to verify.

Proposition 4 Let $\boxplus$ be a compound modality of $\mathcal{F}_{\kappa}$. Then there exists a finite set $S \subseteq \kappa^{*}$ such that $\boxplus p \leftrightarrow \square^{S} p \in \boldsymbol{K}_{K}$.

Fix a normal modal logic $L$. Let $\Delta$ be a set of formulae. We wish to describe the sets $\Delta^{\vdash}:=\left\{\varphi: \Delta \vdash_{L} \varphi\right\}, \Delta^{\Vdash}:=\left\{\varphi: \Delta \Vdash_{L} \varphi\right\}$ as well as the set $L \oplus \Delta$. In the first case we have to close under MP, in the second under MP and MN and in the third under MP, the rule MN and substitution. It turns out that these closures can be obtained in a canonical and simple way. Denote by $\Delta^{s}$ the closure of $\Delta$ under substitution, by $\Delta^{p}$ the closure of $\Delta$ under MP, and by $\Delta^{n}$ the closure of $\Delta$ under MN. 
Proposition 5 The following holds.
1. $\Delta \vdash_{L} \varphi$ iff $\varphi \in(\Delta \cup L)^{p}$
2. $\Delta \Vdash_{L} \varphi$ iff $\varphi \in\left(\Delta^{n} \cup L\right)^{p}$
3. $\varphi \in L \oplus \Delta$ iff $\varphi \in\left(\left(\Delta^{s}\right)^{n} \cup L\right)^{p}$

Proof. The first is immediate from the definition. The second follows from the fact that applications of $\mathrm{MN}_{j}$ can be put before all applications of MP. Moreover, $L$ is closed under MN. The third fact follows from the fact that substitutions commute with $\mathrm{MN}_{j}$ and MP (and that $L$ is closed under $\mathrm{MN}_{j}$ and substitutions). We shall show this now. The proof is based on the notion of a proof tree (rather than a sequence).

Consider an application of $\mathrm{MN}_{j}$ which follows an application of MP as in the left hand side below. There is an alternative proof of $\square_{j} \psi$ in which the order is reversed. This proof is shown to the right.
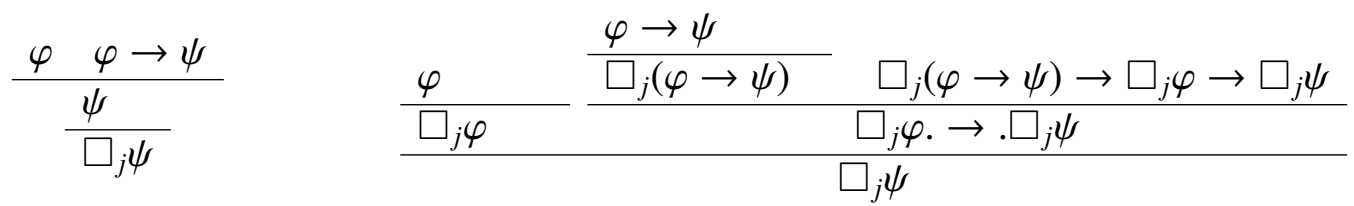

It is readily computed that the depth of the application of $\mathrm{MN}_{j}$ is reduced. A proper inductive argument will establish that each proof can be transformed into a proof where all applications of $\mathrm{MN}_{j}$ precede all applications of MP. It follows that we have $\Delta \vdash_{L} \varphi$ iff $\varphi \in\left((\Delta \cup L)^{n}\right)^{p}=\left(\Delta^{n} \cup L^{n}\right)^{p}=\left(\Delta^{n} \cup L\right)^{p}$. For the rules MN are unary and so $(\Delta \cup L)^{n}=\Delta^{n} \cup L^{n} . L^{n}=L$, since $L$ is normal.

Next we look at substitution. It is easy to show that each application of substitution can be moved up in the proof tree, so that the proof can be arranged in such a way that all applications of substitution precede all applications of $\mathrm{MN}$, which in turn precede all applications of MP. Therefore, $\varphi \in L \oplus \Delta$ iff $\varphi \in\left(\left(\Delta^{s}\right)^{n} \cup\left(L^{s}\right)^{n}\right)^{p}=\left(\left(\Delta^{s}\right)^{n} \cup L\right)^{p}$, by similar arguments.

\section{Global Reduction and Local Reduction}

Let $L$ and $M$ be normal modal logics and $L \subseteq M$. Then also $\Vdash_{L} \subseteq \Vdash_{M}$. Moreover, $M=L \oplus \Sigma$ for some set of formulae $\Sigma$. Then the following is easily shown. 
Lemma 6 Suppose that $M=L \oplus \Sigma$ and $\Delta \Vdash_{M} \varphi$. Then there exists a finite set $\Xi \subseteq \Sigma^{s}$ such that $\Delta ; \Xi \Vdash_{L} \varphi$.

Proof. We use Proposition 5. $M=\left(L \cup\left(\Sigma^{s}\right)^{n}\right)^{p}$. Now $\Delta \Vdash_{M} \varphi$ iff $\varphi \in\left(\Delta^{n} \cup M\right)^{p}$ iff $\varphi \in\left(\Delta^{n} \cup\left(\Sigma^{s}\right)^{n} \cup L\right)^{p}$ iff $\varphi \in\left(\left(\Delta \cup \Sigma^{s}\right)^{n} \cup L\right)^{p}$ iff $\Delta ; \Xi \vdash_{L} \varphi$ for some finite $\Xi \subseteq \Sigma^{s}$.

Of course, $\Xi$ depends on $\Delta$ and $\varphi$. Therefore, we regard $\Xi$ as a function $X$ mapping sets of formulae to sets of theorems of $M$. (More precisely, we should construct $X$ as a function from pairs $\langle\Delta, \varphi\rangle$ to sets of formulae. However, in practice it is enough to assume $X$ to be a function that takes the set $\Delta ; \varphi$ rather than the pair $\langle\Delta, \varphi\rangle$ as its argument. Nothing of substance is lost.) We can also assume that the variables occurring in $X(\Delta)$ are also variables of $\Delta$. For let $\sigma$ be a substitution such that $\sigma(p):=p$ if $p$ occurs in $\Delta$ or $\varphi$ and $\sigma(p):=\perp$ else. Then if $\Delta ; X(\Delta ; \varphi) \Vdash_{L} \varphi$ then also $\Delta^{\sigma} ; X(\Delta ; \varphi)^{\sigma} \Vdash_{L} \varphi^{\sigma}$, which is $\Delta ; X(\Delta ; \varphi)^{\sigma} \Vdash_{L} \varphi$. Since $X(\Delta) \subseteq \Sigma^{s}$, also $X(\Delta ; \varphi)^{\sigma} \subseteq \Sigma^{s}$.

Proposition 7 Let $M=L \oplus \Sigma$. Then there exists a function $X: \wp\left(\mathcal{F}_{\kappa}\right) \rightarrow \Sigma^{s}$ such that

1. $X(\Delta ; \varphi)$ is finite for any $\Delta$.

2. $\operatorname{var}[X(\Delta)] \subseteq \operatorname{var}[\Delta]$

3. $\Delta \Vdash_{M} \varphi$ iff $\Delta ; X(\Delta ; \varphi) \Vdash_{L} \varphi$.

Such a function is called a global reduction function of $M$ to $L$.

Definition 8 Let $L$ be a normal modal logic. L is globally decidable if there is an algorithm computing the answer of the problem ' $\Delta \Vdash_{L} \varphi$ '. L has the global finite model property if $\Delta \Vdash_{L} \varphi$ iff for all finite global L-models $\mathfrak{N}$ : if $\mathfrak{N} \vDash \Delta$ then $\mathfrak{N}=\varphi$.

The following is known as Harrop's Theorem.

Proposition 9 Let L be a a finitely axiomatizable logic. If L has the (global) finite model property is also (globally) decidable.

Proof. Since $L$ is finitely axiomatizable, its set of theorems is recursively enumerable. Its set of nontheorems is enumerated as follows. Since $L$ is finitely axiomatizable, there is a procedure to check whether a finite Kripke-frame is an $L$-frame. 
Thus the set of $L$-frames is also recursively enumerable. It is now straightforward to see that the set of local models is r. e. and thus the set of nontheorems.

For the purpose of the next definition a function from sets to finite sets is computable if its restriction to the set of finite sets is a computable function.

Definition 10 Let $M$ and $L$ be normal modal logics. $M$ is globally constructively reducible to $L$ if there exists a computable global reduction function of $M$ to $L$.

Proposition 11 Suppose that L is globally decidable and $M$ is globally constructively reducible to L. Then $M$ is globally decidable as well.

Proof. Let $\Delta$ and $\varphi$ be given. We describe an algorithm for deciding ' $\Delta \Vdash_{M} \varphi$ '. First, compute $X(\Delta ; \varphi)$ and then compute the answer to the problem ' $\Delta ; X(\Delta ; \varphi) \Vdash_{L}$ $\varphi$ '. This is decidable by assumption. The answer to the latter is the answer to the problem ' $\Delta \Vdash_{M} \varphi$ '.

From this theorem the following can be deduced.

Proposition 12 Suppose that $L$ is globally decidable. Then there exists a computable global reduction function to any logic contained in $L$.

Proof. Let $M \subseteq L, \Delta$ and $\varphi$ be given. We have an algorithm deciding whether or not $\Delta \Vdash_{L} \varphi$. Suppose that $\Delta \Vdash_{L} \varphi$ does not hold. Then put $X(\Delta ; \varphi):=\varnothing$. Then clearly $\Delta ; X(\Delta ; \varphi) \Vdash_{M} \varphi$ also does not hold. Suppose now that $\Delta \Vdash_{L} \varphi$ holds. Then start enumerating all global $L$-proofs starting with $\Delta$. (This is possible since $L$ is decidable. For a global $L$-proof is a sequence such that every element is either a member of $\Delta$, which is decidable since $\Delta$ is finite, a member of $L$, which is decidable by the decidability of $L$, of follows from previous members of the sequence by application of MN or MP.) There is a proof $\Pi$ for $\varphi$ in this list. Let $X(\Delta ; \varphi)$ be the set of all $\chi \in L$ occurring in $\Pi$. Then $\Pi$ is a proof of $\varphi$ from $\Delta ; X(\Delta ; \varphi)$ using MN and MP. Hence $\Delta ; X(\Delta ; \varphi) \Vdash_{M} \varphi$.

Hence, the mere existence of computable global reduction functions is not an exciting fact. More interesting are the upper bounds for the size of such sets. Notice that in the previous theorem $M$ could even be a globally undecidable logic.

Theorem 13 Let $M$ be an extension of $L$ by means of finitely many variable free formulae. Then $M$ is globally constructively reducible to $L$.

Proof. By assumption, $M=L \oplus \Sigma$ for some finite set $\Sigma$ of constant formulae. Notice that $M=(L \cup \Sigma)^{n p}=\left(L \cup \Sigma^{n}\right)^{p}$, since $\Sigma$ is closed under substitution. Put 
$X(\Delta ; \psi):=\Sigma$. Then we have $\Delta \Vdash_{M} \psi$ iff there is a global proof of $\psi$ from $\Delta \cup M$ iff there is a global proof of $\psi$ from $\Delta \cup L \cup \Sigma$ iff there is a global proof of $\psi$ from $\Delta \cup X(\Delta ; \psi) \cup L$ iff $\Delta ; X(\Delta ; \psi) \Vdash_{L} \psi$.

Notice that the theorem is false if $\Sigma$ is infinite. For there exists an infinite set $C$ of independent constant formulae over $\mathbf{K}$. Let $\Sigma$ be a nonrecursive subset of $C$. Then $\mathbf{K} \oplus \Sigma$ is globally undecidable, as is easily seen.

Consider the following functions.

$$
\begin{array}{ll}
X_{4}(\Delta) & :=\{\square \chi \rightarrow \square \square \chi: \square \chi \in s f[\Delta]\} \\
X_{T}(\Delta) & :=\{\square \chi \rightarrow \chi: \square \chi \in s f[\Delta]\} \\
X_{B}(\Delta) & :=\{\neg \chi \rightarrow \square \neg \square \chi: \square \chi \in s f[\Delta]\} \\
X_{G}(\Delta) & :=\{\neg \square \chi \rightarrow \neg \square(\chi \vee \neg \square \chi): \square \chi \in s f[\Delta]\} \\
X_{G r z}(\Delta) & :=\{\neg \square \chi \rightarrow \neg \square(\chi \vee \neg \square(\chi \rightarrow \square \chi)): \square \chi \in \operatorname{sf}[\Delta]\} \\
X_{\text {alt } 1}(\Delta) & :=\{\neg \square \chi \rightarrow \square \neg \chi: \square \chi \in s f[\Delta]\}
\end{array}
$$

The reader may check that the formulae are indeed axioms. We give proofs of the fact that these are reduction functions to $\mathbf{K}$.

Theorem 14 Suppose that L has the global finite model property and that the class of finite L-frames is closed under replacement of $\triangleleft$ by its transitive closure. Then $X_{4}$ is a global reduction function of L.4 to L. In particular, $X_{4}$ is a global reduction function of $\boldsymbol{K}_{4}$ to $\boldsymbol{K}$.

Proof. We have to show that

$$
\Delta \Vdash_{L .4} \varphi \quad \Leftrightarrow \quad \Delta ; X_{4}(\Delta ; \varphi) \Vdash_{L} \varphi
$$

From right to left is straightforward. From left to right, assume $\Delta ; X_{4}(\Delta ; \varphi) \Vdash_{L} \varphi$ is not the case. Then there exists a finite $L$-model $\langle\mathfrak{F}, \beta, x\rangle, \mathfrak{F}=\langle F, \triangleleft\rangle$ such that $\langle\mathfrak{F}, \beta\rangle \vDash \Delta ; X_{4}(\Delta ; \varphi)$ but $\langle\mathfrak{F}, \beta, x\rangle \not \models \varphi$. Let $\triangleleft$ be the transitive closure of $\triangleleft$ and $\mathfrak{F}^{4}:=\langle\mathfrak{F}, \triangleleft\rangle$. By assumption on $L, \mathfrak{F}^{4}$ is a $L$-frame. $\langle F, \triangleleft\rangle$ is transitive; therefore $\mathfrak{F}^{4}$ is a $L .4$-frame. We show that for all subformulae $\chi$ of $\Delta$ or $\varphi$ and all worlds $y$

$$
\langle F, \varangle, \beta, y\rangle \vDash \chi \quad \Leftrightarrow \quad\langle F, \triangleleft, \beta, y\rangle \vDash \chi
$$

This then establishes $\langle F, \boldsymbol{\triangleleft}, \beta\rangle \vDash \Delta$ and $\langle F, \varangle, \beta, x\rangle \vDash \neg \varphi$. We show ( $\dagger$ ) by induction on $\chi$. For variables there is nothing to show. The steps for $\neg$ and $\wedge$ are straightforward. Now let $\chi=\square \chi^{\prime}$. Assume $\langle F, \varangle, \beta, y\rangle \not \models \square \chi^{\prime}$. Then there is a $z$ such that $y \triangleleft z$ and $\langle F, \varangle, \beta, z\rangle \vDash \neg \chi^{\prime}$. By induction hypothesis, $\langle F, \triangleleft, \beta, z\rangle \vDash \neg \chi^{\prime}$. By definition of $\triangleleft$ there is a chain $y=y_{0} \triangleleft y_{1} \triangleleft \ldots \triangleleft y_{n}=z$. Now $\left\langle F, \triangleleft, \beta, y_{n-1}\right\rangle \vDash$ 
$\neg \square \chi^{\prime}$. If $n-1>0$ then $\left\langle F, \triangleleft, \beta, y_{n-2}\right\rangle \vDash \neg \square \square \chi^{\prime}$. Since $\square \chi^{\prime} \rightarrow \square \square \chi^{\prime} \in X_{4}(\Delta ; \varphi)$ and $\left\langle F, \triangleleft, \beta, y_{n-2}\right\rangle \vDash X_{4}(\Delta ; \varphi)$ we must have $\left\langle F, \triangleleft, \beta, y_{n-2}\right\rangle \vDash \neg \square \chi^{\prime}$. Iterating this argument we get $\langle F, \triangleleft, \beta, y\rangle \vDash \neg \square \chi^{\prime}$. So, $\langle F, \triangleleft, \beta, y\rangle \not \models \square \chi^{\prime}$. Clearly, if $\langle F, \triangleleft, \beta, y\rangle \not \models \square \chi^{\prime}$ then $\langle F, \varangle, \beta, y\rangle \not \models \square \chi^{\prime}$, since $\triangleleft \subseteq<$

Theorem 15 Suppose that L has the global finite model property and that the class of finite $L-$ frames is closed under replacement of $\triangleleft$ by its reflexive closure. Then $X_{T}$ is a global reduction function of L.T to L. In particular, $X_{T}$ is a global reduction function of $\boldsymbol{K}$. $\boldsymbol{T}$ to $\boldsymbol{K}$.

Proof. Suppose that we have an $M$-frame $\mathfrak{F}=\langle F, \triangleleft\rangle$ and $\langle\mathfrak{F}, \beta\rangle \vDash X_{T}(\Delta ; \varphi)$. Let $\mathfrak{F}^{T}$ be obtained by replacing $\triangleleft$ by its reflexive closure, $\triangleleft$. By definition, $\mathfrak{F}^{T} \vDash M$ and so $\mathfrak{F}^{T} \vDash M$.T. By induction on the set $s f[\Delta ; \varphi]$ we show that for all $w$ in the transit of $x$

$$
\left\langle\mathfrak{F}^{T}, \beta, w\right\rangle \vDash \chi \quad \Leftrightarrow \quad\langle\mathfrak{F}, \beta, w\rangle \vDash \chi
$$

The only critical step is $\chi=\square \tau$. From left to right this follows from the fact that if $x \triangleleft y$ then also $x \triangleleft y$. For the other direction, assume we have $\left\langle\mathfrak{F}^{T}, \beta, w\right\rangle \not \models \square \tau$. Then there is a $v$ such that $w \triangleleft v$ and $\left\langle\mathfrak{F}^{T}, \beta, v\right\rangle \vDash \neg \tau$. If $v \neq w$, we are done for then also $w \triangleleft v$. So assume the only choice for $v$ is $v=w$ and that $w \nless w$. Then we have $\langle\mathfrak{F}, \beta, w\rangle \vDash \square \tau$. But $\langle\mathfrak{F}, \beta, w\rangle \vDash \square \tau \rightarrow \tau$, by choice of the reduction function. Hence $\langle\mathfrak{F}, \beta, w\rangle \vDash \tau$, and so by induction hypothesis $\left\langle\mathfrak{F}^{T}, \beta, w\right\rangle \vDash \tau$, which is a contradiction. So there always is a successor $v \neq w$.

The proof for $\mathbf{B}$ is as in Theorem 20, so we will omit it here. Clearly, the reduction functions given above work also for polymodal logics under the conditions stated for the logic and for $\triangleleft$, where $\triangleleft$ is replaced by any of the operators. Furthermore, if $X_{2}$ is a reduction function from $L_{2}$ to $L_{1}$, and $X_{1}$ is a reduction function from $L_{1}$ to $L_{0}$ then $X_{2} \circ X_{1}$ is a reduction function from $L_{2}$ to $L_{0}$. So, reductions may applied in succession. We apply this to the following theorem.

Definition 16 A $\kappa$-modal logic $L$ is called an $\boldsymbol{R S T - l o g i c}$ if $L=K \oplus A$ for some $A \subseteq\left\{p \rightarrow \diamond_{j} p, p \rightarrow \square_{j} \diamond_{j} p, \diamond_{j} \diamond_{j} \rightarrow \diamond_{j} p: j<\kappa\right\}$.

Theorem 17 Let L be a finitely axiomatizable RST-logic Then L has the global finite model property.

Proof. Apply the constructive reduction in iteration. Since there are no interaction postulates for the operators, we may add the axioms for each operator independently. For a single operator the result follows from the following observations. 
Let $R$ be a relation. If $R$ is reflexive, so is its symmetric closure and also its transitive closure. If $R$ is reflexive and symmetric, so is its transitive closure.

This result actually follow from the general transfer results of [8], but the proof offered here is much simpler. Now we turn to $\mathbf{G}, \mathbf{G r z}$ and $\mathbf{a l t}_{1}$. Both $\mathbf{G}$ and Grz are transitive logics. We will now show that the functions above establish a reduction from $L . \mathbf{G}$ to $L . \mathbf{K} \mathbf{4}$ under certain conditions and a reduction from $L . \mathbf{G r z}$ to $L . \mathbf{S} 4$ under certain analogous conditions. The first result is a generalization of a theorem by Balbiani and Herzig in [1]. To state it in full generality, we shall introduce the notion of a subframe logic. Let $\langle F, R, U\rangle$ be a $\kappa$-frame, and $G \in U$. Then put $S(j):=R(j) \cap F^{2}, V:=\{A \subseteq F: A \subseteq U\}$. The pair $\langle G, S, V\rangle$ is called a subframe of $\mathfrak{F}$. A logic $L$ is a subframe logic if its class of frames is closed unter taking subframes. By a well-known theorem of Fine [3], $L$ has the finite model property (but see also the appendix for a proof using constructive reduction). The following is proved without this assumption, however.

Theorem 18 Let $L \supseteq K_{4}$ be a subframe logic with the finite model property. Assume that the class of finite L-Kripke frames is closed under replacement of a reflexive point by an irreflexive point. Then $X_{G}$ is a global constructive reduction of L.G to L. In particular, it is a global constructive construction of $\boldsymbol{G}$ to $\mathbf{K} 4$.

Proof. Notice that $L$ is transitive, so we only need to consider reductions where the antecedent is identical to $T$. From this follows the global finite model property of $L$. Put $\square^{+} \varphi:=\varphi \wedge \square \varphi$. Now let $\mathfrak{F}$ be a finite transitive frame and

$$
\left\langle\mathfrak{F}, \beta, w_{0}\right\rangle \vDash \varphi ; \square^{+}\{\neg \square \chi \rightarrow \neg \square(\chi \vee \neg \square \chi): \square \chi \in \operatorname{sf}(\varphi)\}
$$

Now, pick points from the frame as follows. Put $S_{0}:=\left\{w_{0}\right\}$. The sets $S_{n}$ are now defined inductively. Let $x \in S_{n}$ and $\square \chi \in s f(\varphi)$ such that $\langle\mathfrak{F}, \beta, x\rangle \not \models \square \chi$, and no successor of $x$ in $S_{n}-\left\{w_{0}\right\}$ exists such that $\langle\mathfrak{F}, \beta, y\rangle \vDash \neg \chi$. Then, by assumption on the reduction function, $\langle\mathfrak{F}, \beta, x\rangle \vDash \backslash(\neg \chi \wedge \square \chi)$. Hence there exists an $\widehat{x}$ such that $\widehat{x} \vDash \neg \chi ; \square \chi$. (Moreover, if $x=w_{0}$, then $\widehat{x} \neq w_{0}$. For $\widehat{x}$ is irreflexive, and so $w_{0} \triangleleft \widehat{x}$ implies $w_{0} \neq \widehat{x}$.) It follows that $\widehat{x}$ is irreflexive. Put $S_{n+1}:=S_{n} \cup\{\widehat{x}\}$. The selection ends after some steps, since $F$ is finite. Call the resulting set $G$. Let $\triangleleft^{G}:=\triangleleft \cap(G \times G)-\left\{\left\langle w_{0}, w_{0}\right\rangle\right\}$. Then put $\mathfrak{G}:=\left\langle G, \triangleleft^{G}\right\rangle$. (Alternatively, we might simply take $\mathfrak{G}$ to be the subframe consisting of $w_{0}$ and all irreflexive points from $\mathfrak{F}$, with the transition $w_{0} \rightarrow w_{0}$ being removed.) $\mathfrak{G}$ is transitive and irreflexive, hence it is a frame for $\mathbf{G}$. Since $L$ is a subframe logic and closed under changing a reflexive point into an irreflexive point, $\mathfrak{G}$ is also a frame for $L$. Put $\gamma(p):=\beta(p) \cap G$. We now show that for every subformula $\psi$ of $\varphi$ and every point 
$y \in G,\langle\mathfrak{G}, \gamma, y\rangle \vDash \psi$ iff $\langle\mathfrak{F}, \beta, y\rangle \vDash \psi$. This holds for variables by construction, and the steps $\neg, \wedge$ are straightforward. Now suppose $\langle\mathfrak{G}, \gamma, y\rangle \not \models \square \chi$. Then also $\langle\mathfrak{F}, \beta, y\rangle \not \models \square \chi$. Conversely, suppose that $\langle\mathfrak{F}, \beta, y\rangle \not \models \square \chi$, for some $\square \chi \in \operatorname{sf}(\varphi)$. Then also $\langle\mathfrak{G}, \beta, y\rangle \not \models \square \chi$, since a successor $z$ for $y$ has been chosen such that $\langle\mathfrak{F}, \beta, z\rangle \vDash \chi ; \square \neg \chi$. By induction hypothesis, $\langle\mathfrak{G}, \gamma, z\rangle \vDash \chi$. Moreover, $y \triangleleft^{G} z$. For if $y \neq w_{0}$ this holds by definition of $\triangleleft^{G}$. For $y=w_{0}$ observe that either $w_{0} \triangleleft^{F} w_{0}$, and then $z \neq w_{0}$, since $z \Varangle z$. From this follows $w_{0} \triangleleft^{G} z$. Or else, $w_{0} \Varangle^{F} w_{0}$, in which case $w_{0} \triangleleft^{G} z$ anyway. And so $\langle\mathfrak{G}, \gamma, y\rangle \not \models \square \chi$, as required.

Theorem 19 Let L be a subframe logic containing S4. Assume that L has the finite model property. Then $X_{G r z}$ is a constructive global reduction function of L.Grz to L.

Proof. $L$ has the (global) finite model property. Let $\left\langle\mathfrak{F}, \beta, w_{0}\right\rangle$ a finite $\mathbf{S 4 - m o d e l}$ such that

$$
\left\langle\mathfrak{F}, \beta, w_{0}\right\rangle \vDash \varphi ; \square^{+}\{\neg \square \chi \rightarrow \neg \square(\chi \vee \neg \square(\chi \rightarrow \square \chi)): \square \chi \in s f(\varphi)\} .
$$

(Here, again $\square^{+} \varphi:=\varphi \wedge \square \varphi$.) We select a subset $G$ of $F$ in the following way. We start with the set $S_{0}:=\left\{w_{0}\right\}$. $S_{n+1}$ is defined inductively as follows. Suppose $x \in$ $S_{n}$ and $\langle\mathfrak{F}, \beta, x\rangle \vDash \neg \square \chi$, but no $y$ exists in $S_{n}$ such that $x \triangleleft y$ and $\langle\mathfrak{F}, \beta, y\rangle \vDash \neg \chi$. We choose a successor $y$ of $x$ such that $y \vDash \neg \chi ; \square(\chi \rightarrow \square \chi)$ and put $S_{n+1}:=S_{n} \cup\{y\}$. $y$ exists by choice of the reduction function. Now the following holds. (Recall that in S4-frames, sets of the form $C(x):=\{y: x \triangleleft y \triangleleft x\}$ are called clusters. See also Section 8.) (a) The entire cluster $C(y)$ satisfies $\neg \chi$, (b) no point in a cluster succeeding $C(y)$ and different from $C(y)$ satisfies $\chi$. This procedure comes to a halt after finitely many steps. The resulting set is called $G$, and the subframe based on it $\mathfrak{G}$. It is directly verified that $G$ contains at most one point from each cluster. (Moreover, the selection procedure produces a model whose depth is bounded by the number of formulae in $s f(\varphi)$ of the form $\square \chi$ as can easily be seen.) So all clusters have size $1 . \mathfrak{G}$ is reflexive and transitive, being a subframe of $\mathfrak{F}$. So, $\mathfrak{G}$ is a Grz-frame. Since $L$ is a subframe logic, $\mathfrak{G}$ is a $L$-frame as well. Let $\gamma(p):=\beta(p) \cap G$. It is shown as in the previous proof that for every subformula $\chi$ of $\varphi$ and every $x \in G,\langle\mathfrak{G}, \gamma, x\rangle \vDash \chi$ exactly when $\langle\mathfrak{F}, \beta, x\rangle \vDash \chi$. In particular, $\left\langle\mathfrak{G}, \gamma, w_{0}\right\rangle \vDash \varphi$. This concludes the proof.

So far all the axioms have been unimodal. Here now is an example of a bimodal axiom. Axioms of this form are used to axiomatize tense logic. The axiom of symmetry is also of this form (except that there is only one modality, not two). If $R$ is a binary relation, we shall write $R^{\smile}$ to denote the converse of $R$, i. e. the set $\{\langle y, x\rangle: x R y\}$. 
Theorem 20 Let L be a bimodal logic that has the global finite model property. Assume that the class of finite L-Kripke frames is closed under passing from $\left\langle F, \triangleleft_{0}, \triangleleft_{1}\right\rangle$ to $\left\langle F, \triangleleft_{0}, \triangleleft_{1} \cup \triangleleft_{0}^{\smile}\right\rangle$. Put

$$
X_{01}(\Delta):=\left\{\neg \chi \rightarrow \square_{0} \neg \square_{1} \chi: \square_{0} \chi \in s f[\Delta]\right\}
$$

Then $X_{01}$ globally reduces $L \oplus p \rightarrow \square_{0} \triangleright_{1} p$ to $L$.

Proof. Let $L(01):=L \oplus p \rightarrow \square_{0} \searrow_{1} p$. We show that

$$
\Delta \Vdash_{L(01)} \varphi \quad \Leftrightarrow \quad \varphi ; X_{01}(\Delta ; \psi) \Vdash_{L} \varphi
$$

Let $\mathfrak{M}=\left\langle\mathfrak{F}, \beta, w_{0}\right\rangle$ be a local model where $\mathfrak{F}=\left\langle F, \triangleleft_{0}, \triangleleft_{1}\right\rangle$ is a finite $\mathbf{K}_{2}$-frame such that $\langle\mathfrak{F}, \beta\rangle \vDash \Delta ; X_{01}(\Delta ; \varphi)$ and $\left\langle\mathfrak{F}, \beta, w_{0}\right\rangle \vDash \neg \varphi$. Let $\triangleleft_{0}:=\triangleleft_{0}$ and $\triangleleft_{1}:=\triangleleft_{1} \cup \triangleleft_{0}^{\longleftarrow}$. Then $\left\langle F, \varangle_{0}, \triangleleft_{1}\right\rangle$ is a $L(01)$-frame, for it is a $L$-frame by assumption on $L$; and $\triangleleft_{1} \supseteq \triangleleft_{0}^{\smile}$. For all $\chi \in s f[\Delta ; \varphi]$ we have

$$
\left\langle F, \triangleleft_{0}, \triangleleft_{1}, \beta, y\right\rangle \vDash \chi \quad \Leftrightarrow \quad\left\langle F, \triangleleft_{0}, \triangleleft_{1}, \beta, y\right\rangle \vDash \chi .
$$

This is clear for variables; the steps for $\neg$ and $\wedge$ are straightforward. Likewise the step for $\chi=\square_{0} \tau$. Now let $\chi=\square_{1} \tau$. From left to right is clear. Now right to left; assume $\left\langle F, \boldsymbol{\iota}_{0}, \boldsymbol{\iota}_{1}, \beta, y\right\rangle \not \forall \square_{1} \tau$. Then there is a $w$ such that $y \boldsymbol{\iota}_{1} w$ and $\left\langle F, \triangleleft_{0}, \triangleleft_{1}, \beta, w\right\rangle \vDash \neg \tau$. By induction hypothesis, $\left\langle F, \triangleleft_{0}, \triangleleft_{1}, \beta, w\right\rangle \vDash \neg \tau$. If $y \triangleleft_{1} w$, we are done; for then $\left\langle F, \triangleleft_{0}, \triangleleft_{1}, \beta, y\right\rangle \not \models \square_{0} \tau(=\chi)$. Otherwise $w \triangleleft_{0} y$. Now, $\left\langle F, \triangleleft_{0}, \triangleleft_{1}, \beta, w\right\rangle \vDash \square_{0} \neg \square_{1} \tau$, since $\left\langle F, \triangleleft_{0}, \triangleleft_{1}, \beta, w\right\rangle \vDash X_{01}(\Delta ; \varphi)$. Thus $\left\langle F, \triangleleft_{0}, \triangleleft_{1}, y\right\rangle \vDash$ $\neg \square_{1} \tau$. So, $\left\langle F, \triangleleft_{0}, \triangleleft_{1}, \beta, y\right\rangle \not \models \square_{1} \tau$.

Before we prove some more results in this vein, let us indicate something about the scope and the limits of this technique. Suppose that we have a postulate that is elementary on all finite frames, and that the condition on finite frames is a universal, positive restricted sentence. That is, it is of the form $\forall x . \alpha(x)$ where $\alpha(x)$ is made from statements $x \triangleleft_{j} y, j<\kappa$, and $x \doteq y$, using the connectives $\wedge$ and $\vee$ and

$$
\left(\forall y \triangleright_{j} x\right) \beta:=(\forall y)\left(x \triangleleft_{j} y \rightarrow \beta\right)
$$

Then $\alpha$ is a Sahlqvist condition and corresponds to a modal formula $\varphi_{\alpha}$. More precisely, if $\alpha=Q \vec{x} . \Phi(\vec{x})$, where $\Phi$ is quantifier free, we can define for each disjunct $\delta$ of $\Phi$ a formula $\mu_{\delta}$ such that

$$
\varphi_{\alpha}=\sigma \rightarrow \bigvee \mu_{\delta}
$$

Here, $\sigma$ and the $\mu_{\delta}$ are formulae made from of variables using $\wedge, \vee$ and $\nabla_{j}, j<\kappa$. Let $\alpha$ not contain any occurrences of $x \doteq y$. Then we can view the elementary 
property $\alpha$ as a closure condition on the accessibility relations. If a certain tree can be mapped homomorphically into the frame, then some more relations must hold. The tree is actually defined by the variables of the restricted quantifiers plus the restrictions that apply to them. We call this the carrier tree of $\alpha$. For example, transitivity is $(\forall x)(\forall y \triangleright x)(\forall z \triangleright y)(x \triangleleft z)$. So, if the tree $x \triangleleft y \triangleleft z$ can be mapped into the frame (not necessarily injectively) then $x \triangleleft z$ must obtain as well. Likewise for alt $_{1}$, which is $(\forall x)(\forall y \triangleright x)(\forall z \triangleright x)(y \doteq z)$. If $\mathfrak{F}$ and $\mathfrak{G}$ are frame such that $F=G$ and $\triangleleft_{j}^{\mathfrak{F}} \subseteq \triangleleft_{j}^{\mathfrak{G}}$ for all $j<\kappa$ then we say that $\mathfrak{G}$ is an arrow extension of $\mathfrak{F}$. We may define for a frame $\mathfrak{F}$ the set $C^{\alpha}(\mathfrak{F})$ to be the set of all minimal arrow extensions $\mathfrak{G}$ of $\mathfrak{F}$ such that $\mathfrak{G} F(\forall x) \alpha(x)$. Then if $L$ has the finite model property and $\alpha$ is elementary on the finite frames, and it holds that for all finite $L$-frames $\mathfrak{F}, C^{\alpha}(\mathfrak{F})$ is a set of $L$-frames it seems that $L \oplus \varphi_{\alpha}$ has the finite model property, and that this can be shown by means of the global reduction sets.

We are not able to provide a proof for that claim, in fact we are not sure whether the claim holds in full generality. However, it might be worthwile to explain the idea of a proof that works quite well in many concrete cases. It is roughly as follows. Take $\Delta$ and $\varphi$ such that $\varphi$ does not follow globally from $\Delta$ in $L \oplus \varphi_{\alpha}$. Define $Y(\Delta)$ to be the set of those instances of $\varphi_{\alpha}$, where conjunction of subformulae of $\Delta$ or their negations are substituted for the variables. This is a finite set, and $\operatorname{var}[Y(\Delta)] \subseteq \operatorname{var}[\Delta]$. We need to show that if there is a $L$-model $\mathfrak{M}=\langle\mathfrak{F}, \beta\rangle$ such that $\mathfrak{M} \vDash \Delta ; Y(\varphi ; \Delta)$ but $\mathfrak{M} \not \models \varphi$, then we define a closure $\mathfrak{G} \in C^{\alpha}(\mathfrak{F})$ in the following way. Suppose that the carrier tree of $\alpha$ is embeddable, say $x \mapsto w_{x}$. Then $\sigma$ can be made true at the root of the tree. Substitute for the variable $p_{x}$ the conjunction of all subformulae of $\Delta ; \varphi$ that are true at $w_{x}$ and the conjunction of all negations of such formulae which are false at $w_{x}$. (We call this the atom of $\left.w_{x}.\right)$ By force of $Y(\varphi ; \Delta)$ there must be a disjunct $\mu_{\delta}$ of $\varphi_{\alpha}$, which is true. It corresponds to a disjunct $\delta$ of the matrix of $\alpha$. Hence, we add those relations $w_{x} \triangleleft_{j} w_{y}$ for which $x \triangleleft_{j} y$ is a conjunct of $\delta$. After having done so, we need to show that for this newly created model $\mathfrak{M}^{1}$ we have $\mathfrak{M}^{1} \vDash \Delta ; Y(\varphi ; \Delta)$, but $\mathfrak{M}^{1} \not \models \varphi$. (It is here that some more is needed to show this. We do not know how to supply the details in the general case, but for many specific cases it works.) If that is so, we continue the process so long as the $\alpha$ is still not satisfied. When we are done, however, the resulting frame is a member of $C^{\alpha}(\mathfrak{F})$ and so a model for $L \oplus \varphi_{\alpha}$, by assumption. Hence, it defines a global model form $\Delta$ in which $\varphi$ is not satisfied.

A case in point are in addition to $4, \mathbf{T}, \mathbf{X}(\mathbf{0 1})$ and $\mathbf{B}$ also the formulae $(\forall x)\left(\forall y \triangleright_{i}\right.$ $x)\left(x \triangleleft_{j} y\right)$, corresponding to $\diamond_{i} p \rightarrow \diamond_{j} p$. Furthermore, we may add to a $\kappa$-modal logic a new modality whose relation includes a given set $S \subseteq \kappa$ of relations and 
that is either transitive (we call it an $S$-master) or an equivalence relation (then it is an $S$-universal modality, see [6]). Constructive reduction can be applied there as well. Notice that the commutation axioms $\diamond_{i} \diamond_{j} p \rightarrow \diamond_{j} \diamond_{i} p$ do not fall into this class since they do not correspond to a universal formula. The just mentioned axiom corresponds to $(\forall x)\left(\forall y \triangleright_{i} y\right)\left(\forall z \triangleright_{j} y\right)\left(\exists u \triangleright_{j} x\right)\left(u \triangleleft_{i} z\right)$. Indeed, it is known that the logic of three commuting S5-modalities is undecidable (this follows from results in Gabbay and Shehtman [4]). Hence, since the logic S5 $\otimes \mathbf{S 5} \otimes \mathbf{S 5}$ is an RST-logic and therefore has the finite model property, it must be the commutation axioms that lead to undecidability.

Another technique, that works especially well with subframe axioms is that of dropping points from a model. In fact, we show in the Appending to this paper that such a procedure can be used to show that all subframe logics containing K4 have the finite model property. This constitutes a constructive proof of the theorem by Fine ([3]). The proof is in fact quite involved, but not more complex than the original one. Likewise, it is possible to show that $\mathbf{a l t}_{n}$, if added to a subframe logic with the (global) finite model property then the resulting logic has the finite model property again and is a subframe logic. It is well known that all subframe logics containing K.alt ${ }_{n}$ have the local finite model property. Our result is a strengthening for some logics. However, these techniques need to be handled with care. We give the following negative example. Consider the monomodal logic corresponding to the following first-order properties:

$(\forall x, y, z \triangleright w)(x \doteq y \vee x \doteq z \vee y \doteq z), \quad(\forall x, y \triangleright w)(\forall z \triangleright w)(z \triangleright x \vee z \triangleright y \vee x \doteq y)$

This logic is an extension of $\mathbf{K}$. alt $_{2}$. Its frame are such that any point has at most two direct successors and at most three two-step successors. By encoding a tiling problem, [15] has shown that this logic is globally undecidable. This shows that we cannot strengthen the methods to all subframe logics.

\section{Complexity}

In this section we will discuss upper bounds on complexity of modal logics that can be derived using the reduction functions. We will mainly deal with the global complexity, since this is the easiest point of attack. From the global complexity, one can also establish results on the local complexity (using the gl-reduction of next section), but they tend to be far worse than the known bounds. For the results on modal logic complexity we refer here generally to [15] and [2] and references therein, though some specific references are also given below. 
Before we start, we have to discuss the notion of the length of a formula. Standardly, the length of a formula is the number of symbols occurring in it. This length is denoted here by $|\varphi|$. It is defined inductively as follows.

$$
\begin{aligned}
& |\perp|:=1 \\
& \left|p_{i}\right| \quad:=1 \\
& |\neg \varphi| \quad:=1+|\varphi| \\
& |\varphi \wedge \psi|:=1+|\varphi|+|\psi| \\
& \left|\square_{j} \varphi\right|:=1+|\varphi|
\end{aligned}
$$

(The variable $p_{i}$ counts as one symbol, even though it would be more appropriate to code the index by a bit sequence. However, we shall ignore this detail here.) For a set $\Delta$, put $|\Delta|:=\sum_{\varphi \in \Delta}|\varphi|$. The symbol count is however not always the most economical way of writing down a formula, especially when $\Delta$ contains few subformulae. This is the case with the formula sets we are dealing with here. Therefore, we shall work with a different measure, in line with [2]. Namely, for a set $\Delta$ of formulae we put

$$
\sharp(\Delta):=\operatorname{card}(\operatorname{sf}[\Delta])
$$

To get acquainted with this measure, we shall note a few of its properties. The following is proved by induction.

Lemma 21 Suppose that no formula occurs more than once in $\Delta$. Then $|\Delta|=\sharp(\Delta)$.

Proposition $22 \log _{2}(|\Delta|+1) \leq \sharp(\Delta) \leq|\Delta|$. These bounds are sharp.

Proof. Clearly, $\sharp(\Delta) \leq|\Delta|$. Now suppose that $\Delta$ is a set of formulae such that every variable and $\perp$ occur at most once. Then it is not hard to see that each formula occurs in $\Delta$ at most once, and in this case $|\Delta|=\sharp(\Delta)$ by the previous lemma. Now we turn to the other inequality. It is clear that the maximum depth of the formulae in $\Delta$ (in terms of their tree structure) provides a lower bound on the number of subformulae, since no two formulae of different depth can be equal. A formula of depth $n$ can have up to $2^{n}-1$ symbols, as is easily shown. So, $n \geq \log _{2}(|\Delta|-1)$. Now define the following formulae.

$$
\begin{array}{ll}
\chi_{1} & :=\perp \\
\chi_{n+1} & :=\chi_{n} \wedge \chi_{n}
\end{array}
$$

Then $\sharp\left(\chi_{n}\right)=n$, but $\left|\chi_{n}\right|=2^{n}-1$. Thus, $\log _{2}\left(\left|\chi_{n}\right|+1\right)=n=\sharp\left(\chi_{n}\right)$.

Proposition 23 The following holds. 
1. If $\varphi \wedge \varphi \in \Delta$ then $\sharp(\Delta ; \varphi \wedge \psi)=\sharp(\Delta ; \varphi ; \psi)$.

2. If $* \varphi \in \Delta, * \in\left\{\square_{j}: j<\kappa\right\} \cup\{\neg\}$, then $\sharp(\Delta ; * \varphi)=\sharp(\Delta ; \varphi)$.

3. $\sharp(\varphi \rightarrow \psi)=\sharp(\neg(\varphi \wedge \neg \psi))=3+\sharp(\varphi ; \psi)$.

4. $\sharp(\varphi \leftrightarrow \psi)=\sharp((\varphi \rightarrow \psi) \wedge(\psi \rightarrow \varphi))=7+\sharp(\varphi ; \psi)$.

5. $\sharp\left(\Delta ; \Delta^{\prime}\right)=\sharp(\Delta)+\sharp\left(\Delta^{\prime}\right)-\operatorname{card}\left(s f[\Delta] \cap s f\left[\Delta^{\prime}\right]\right)$.

These claims are easy to prove, and we shall make tacit use of them in sequel. We note now the following.

Proposition 24 Let $Y$ be any of the reduction functions of the previous section. Then for any set $\Delta, \sharp(Y(\Delta)) \leq c_{Y} \sharp(\Delta)$, where $c_{Y}>0$ is a constant depending only on $Y$.

We verify this with $X_{4}$. Take $\Delta$. For any $\square \chi \in s f[\Delta], X_{4}(\Delta)$ contains the additional formula $\square \chi \rightarrow \square \square \chi$, which is in fact $\neg(\square \chi \wedge \neg \square \square \chi)$. Now

$$
s f(\square \chi \rightarrow \square \square \chi)=s f(\chi) \cup\{\neg(\square \chi \wedge \neg \square \square \chi), \square \chi \wedge \neg \square \square \chi, \neg \square \square \chi, \square \square \chi, \square \chi\}
$$

It follows that for each subformula of $\Delta, X(\Delta)$ adds 5 more formulae. Hence $\sharp\left(X_{4}(\Delta)\right) \leq 6 \sharp(\Delta)$. (The reader may verify that $\left|X_{4}(\Delta)\right|$ is quadratic in $|\Delta|$.)

Definition 25 Let $X$ is a function from finite sets of formulae to finite sets of formulae. We say that $X$ is linear (polynomial, exponential) if $\sharp(X(\Delta)) \leq f(\sharp(\Delta)$ ) for some linear (polynomial, exponential) function $f: \omega \rightarrow \omega$.

Definition 26 A logic L is globally NP(PSPACE, EXPTIME) if there exists a nondeterministic algorithm taking time polynomial in $\sharp(\Delta ; \varphi)$ (a deterministic algorithm taking space polynomial/exponential in $\sharp(\Delta ; \varphi)$ ) which computes the answer to the problem ' $\Delta \Vdash_{L} \varphi$ '.

Similarly, $L$ is globally NP-hard (globally PSPACE-hard etc.) if some problem that is NP-complete (PSPACE-complete etc.) can be polynomially reduced to a problem of the form ' $\Delta \Vdash_{L} \varphi$ '.

Now suppose that we have a logic $L$ which is Q-hard, where $Q$ is any of the three complexity classes. Let $M$ be globally reducible to $L$ by a linear reduction function $X$. Then the problem ' $\Delta \Vdash_{M} \varphi$ ' is equivalent to the problem ' $\Delta ; X(\Delta ; \varphi) \Vdash_{L} \varphi$ '. The latter takes $O(\sharp(\Delta ; \varphi ; X(\Delta ; \varphi)))=O(\Delta ; \varphi)$ time (space). Hence, the space and time complexity does not rise. 
Theorem 27 Suppose that $X$ is a linear global reduction function from $L$ to $M$. Then if $M$ is globally NP(PSPACE, EXPTIME), so is L.

This method only gives an upper bound. In general, $L$ can have much lower complexity than $M$. A trivial example is the inconsistent logic. A more instructive example is the following. S5 is globally linearly reducible to $\mathbf{K}$, by the results of the preceding section. Yet, $\mathbf{K}$ is globally EXPTIME-complete while S5 is NPcomplete.

Corollary 28 The systems $K B, K T, K B T, K 4, G, S 4, G r z$ are globally in EXPTIME. Moreover, tense logic and RST-logics are in EXPTIME.

This is for the transitive logics mentioned in the theorem not the best possible result. They are all PSPACE-complete. However, such a result would follow by the same techniques, if only it is established that K4 is PSPACE-complete. This last statement, however, has to be established independently. There is a possibility to show this, namely using tableaux. It is known that $\mathbf{K}$ has a tableau calculus (for local consistency) in which the branches have a linear length. Using this tableau calculus one can establish the same property for K4. This means that validity can be checked in polynomial space. The reduction sets can be used to show this. We will not go into the details here. Suffice it to say that the length of branches in a tableau can be bounded from above by a polynomial function (see [14] for some tableau calculi for modal logics).

Now consider again the case where $M$ is globally linearly reducible to $L$. Let $L^{\prime}$ be a logic such that $L \subseteq L^{\prime} \subsetneq M$. Then $M$ is globally linearly reducible to $L^{\prime}$ as well. Hence, the complexity class of $M$ is also bounded from above by the complexity class of $L^{\prime}$. It follows that the complexity class of $M$ is the minimum over all complexity classes over the logics containing $L$ and properly contained in $M$. Moreover, if $M$ is $\mathrm{C}$-hard for some complexity class $\mathrm{C}$, then all logics in the interval $[L, M]$ are $\mathrm{C}$-hard. This allows to establish lower bounds for entire intervals of logics, analogous to the result of Ladner ([10]) that all logics in [K, S4] are locally PSPACE-hard. Unfortunately, using our methods we obtain the same lower bound for global complexity of these logics, since $\mathbf{S 4}$ is globally PSPACEcomplete.

In analogy to Chagrov and Zakharyaschev we define the global complexity function of a logic as follows.

Definition 29 Let $M$ be a modal logic with the global finite model property. Let $f_{M}$ be the following function. $f_{M}(n)=\max \{\mu(\Delta ; \varphi): \sharp(\Delta ; \varphi) \leq n\}$, where $\mu(\Delta ; \varphi)$ is 
0 if $\Delta \Vdash_{M} \varphi$ and else it is the least number $p$ such that there exists a global model $\mathfrak{N}$ based on $p$ worlds such that $\mathfrak{N} \vDash \Delta$ and $\mathfrak{N} \not \models \varphi$.

The local complexity function is defined analogously, where the maximum is taken only over $\mu(\varnothing ; \varphi)$. Chagrov and Zakharyaschev show that the local complexity function can be extremely complex, for example the $k$-fold iteration of the exponential function. However, here we are interested only in whether the complexity function is polynomial or exponential. The following is now clear.

Theorem 30 Suppose that L has a polynomial (exponential) global complexity function and that $M$ is globally reducible to L by means of a linear complexity function. Then $M$ has a polynomial (exponential) global compexity function as well.

Proof. Let $f_{L}$ be the global complexity function of $L$ and $X$ the reduction function of $M$ to $L$. Let $p$ be defined by $p(n):=\max \{\sharp(X(\Delta)): \sharp(\Delta) \leq n\}$. Suppose that $\Delta \nVdash_{M} \varphi$ and $\sharp(\Delta ; \varphi) \leq n$. Then $\Delta ; X(\Delta ; \varphi) \nVdash_{L} \varphi$. By assumption there is a model of size $\leq f_{L}\left(\sharp(\Delta ; \varphi ; X(\Delta ; \varphi)) \leq f_{L}(p(n)+1)\right.$. So, $f_{M}:=f_{L}(p(n)+1)$ is a global complexity function for $M$. If $f_{L}$ and $p$ are both polynomial (exponential), so is $f_{M}$.

Again, these results establish only upper bounds. K does not have a polynomial local complexity function and hence also not a polynomial global complexity function as we shall show below. However, for $\mathbf{S 5}$ the global complexity function is polynomial. Incidentally, we may use a result of Chagrov and Zakharyaschev to derive a lower bound for the global complexity function for $\mathbf{K}$. Namely, it is shown that for the local complexity function for $\mathbf{K} \mathbf{4}, f_{4}$, it holds that $\log _{2} f_{4}(n)$ is linear in the limit. Hence the local complexity function is exponential. It is an easy matter to show that the global complexity function, $g_{4}$, is then also exponential. For $\Delta \Vdash_{\mathbf{K} 4} \varphi$ iff $\Delta ; \square \Delta \vdash_{\mathbf{K} 4} \varphi$, where $g_{4}(n) \leq f_{4}(2 n)$. Moreover, $f_{4}(n) \leq g_{4}(n)$. Now, since $\mathbf{K} \mathbf{4}$ can be reduced globally to $\mathbf{K}$, we obtain that the global complexity function for $\mathbf{K}$ cannot be less than exponential. We denote by $k_{\sharp}$ the global complexity function of $\mathbf{K}_{\kappa}$, and $k_{g}$ the local complexity function.

Lemma $31 k_{\sharp}(n) \leq 2^{n}$.

Proof. Let $\Delta \nVdash \varphi, \Delta$ finite. Then by Theorem 42 there exists a global model $\mathfrak{N}=\langle\mathfrak{F}, \beta\rangle$ based on a Kripke-frame $\mathfrak{F}$ such that $\mathfrak{N} \vDash \Delta$ but $\mathfrak{N} \not \models \varphi$. Now filtrate this model with respect to the set $A:=s f[\Delta ; \varphi]$. That means the following. Write $x \approx_{A} y$ if $\langle\mathfrak{F}, \beta, x\rangle \vDash \chi$ is equivalent to $\langle\mathfrak{F}, \beta, y\rangle \vDash \chi$ for all $\chi \in A$. Now let $[x]_{A}:=\left\{y: x \approx_{A} y\right\}$. Put $[x]_{A} \triangleleft_{j}[y]_{A}$ iff there exist $x^{\prime} \approx_{A} x$ and $y^{\prime} \approx_{A} y$ such that 
Figure 1: The branching Tree

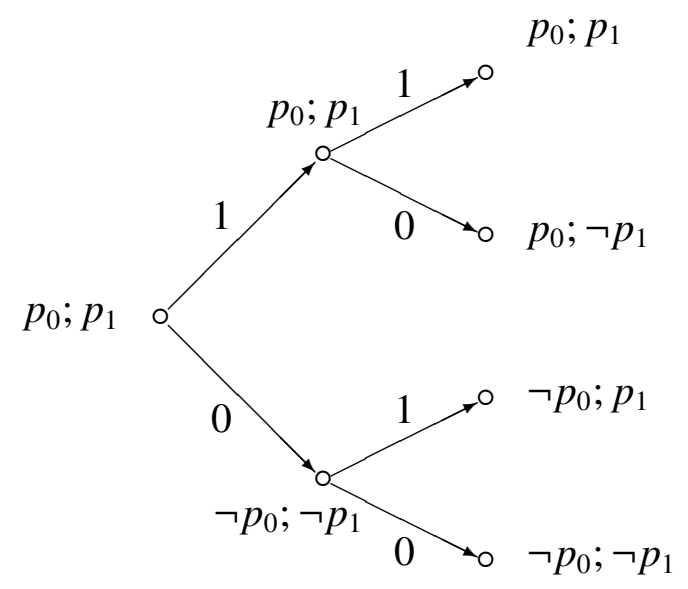

$x^{\prime} \triangleleft_{j} y^{\prime}$. This defines the frame $\mathfrak{F} / A$. Next we put $\beta / A(p):=\left\{[x]_{A}: x \in \beta(p)\right\}$. Then it is shown by induction that $\left\langle\mathfrak{F} / A, \beta / A,[x]_{A}\right\rangle \vDash \chi$ iff $\langle\mathfrak{F}, \beta, x\rangle \vDash \chi$ for all $\chi \in A$. Clearly, $\mathfrak{F} / A$ has at most $2^{n}$ elements, where $n=\sharp(\Delta ; \varphi)$.

Theorem 32 The logics $\boldsymbol{K}_{\kappa}$ have the global finite model property and are globally decidable. Furthermore, the following holds in the limit:

1. $2^{n / 16}<k_{\sharp}(n) \leq 2^{n}$.

2. $2^{\sqrt{n}}<k_{g}(n) \leq 2^{n}$.

Proof. Let $m$ be a natural number. Consider the formulae

$$
\varphi_{i}:=\neg \square^{m-i} \perp . \leftrightarrow . \neg \square p_{i} \wedge \neg \square \neg p_{i}
$$

We claim that

$$
\square^{m+1} \perp ;\left\{\varphi_{i}: i<m\right\} \nVdash \square^{m} \perp \vee \diamond^{m-1} \square \perp
$$

A countermodel is constructed as follows. Let $F$ be the set of at most $m$-long sequences from $\{0,1\}$. Put $\vec{x} \triangleleft \vec{y}$ iff $\vec{y}=\vec{x} a$ for $a \in\{0,1\}$. This defines the frame $\mathfrak{F}$. 
It is rooted at $\varepsilon$ (the empty sequence) and has $2^{m+1}-1$ elements. Furthermore, let $\beta\left(p_{i}\right)$ consist of those $\vec{x}$ such that either (a) $\vec{x}$ has length at least $i+1$ and $\vec{x}=\vec{u} 1 \vec{v}$ for some $\vec{u}$ of length $i$, or (b) $\vec{x}=\varepsilon$, or (c) $\vec{x} \neq \varepsilon, \vec{x}$ has length $\leq i$ and $\vec{x}=\vec{u} 1$ for some $\vec{u}$. (Figure 1 illustrates the model for $m=2$.) Then $\langle\mathfrak{F}, \beta, \varepsilon\rangle \not \models \diamond^{m-1} \square \perp ; \square^{m} \perp$. However, $\langle\mathfrak{F}, \beta\rangle \vDash \square^{m+1} \perp$. Further, if $\vec{x} \models \diamond^{m-i} \top$, then $\vec{x}$ has length at most $i$. Hence $\vec{x} 0 \models \neg p_{i}$ and $\vec{x} 1 \models p_{i}$, from which follows $\vec{x} \models \diamond p_{i} \wedge \diamond \neg p_{i}$. If however $\vec{x} \not \models \diamond^{m-i+1} \mathrm{~T}$ then $\vec{x}$ has length at least $i+1$, and then either (a) $\vec{x}=\vec{u} 0 \vec{v}$ for some $\vec{u}$ of length $i$ or (b) $\vec{x}=\vec{u} 1 \vec{v}$ for some $\vec{u}$ of length $i$. Then $\vec{x} \models \square \neg p_{i}$ in Case (a) and $\vec{x} \mid \square p_{i}$ in Case (b), as is easily seen. Hence $\langle\mathfrak{F}, \beta\rangle \vDash \varphi_{i}$ for every $i<m$. This establishes $(\dagger)$.

Now we show that any model witnessing $(\dagger)$ must have at least $2^{m+1}-1$ points. Suppose that $\langle\mathfrak{G}, \gamma\rangle$ is such a model. Then $\langle\mathfrak{G}, \gamma\rangle \models \Delta$, where $\Delta:=\square^{m+1} \perp ;\left\{\varphi_{i}:<\right.$ $m\}$ and $\langle\mathfrak{G}, \gamma\rangle \not \models \square^{m} \perp \vee \diamond^{m-1} \square \perp$. Then there is an $x \in G$ satisfying $\diamond^{m} \top \wedge \square^{m-1} \diamond \top$. Furthermore, $x \vDash \square^{m+1} \perp$, so any maximal path from $x$ has length $m$, and there exists a path of length $m$. Now, consider a point $x$ of depth $m-i+1$. Then $x \vDash \diamond^{m-i}$ T. If $p_{i}$ holds at $x$, it will hold at every successor of $x$; and if $\neg p_{i}$ holds, it holds at every successor of $x$. However, $p_{i-1}$ holds at one successor and fails at another. So, $x$ has at least two successors. It follows easily that $G$ has least $1+2+2^{2}+2^{n}=2^{m+1}-1$ points.

This allows to prove the upper complexity bound. Let $n$ be given. Then

$$
\begin{aligned}
\sharp\left(\varphi_{i}\right) & =\sharp\left(\neg \square^{m-i} \top \leftrightarrow\left(\neg \square p_{i} \wedge \neg \square \neg p_{i}\right)\right) \\
& =7+\sharp\left(\neg \square^{m-i} \perp ; \neg \square p_{i} \wedge \neg \square \neg p_{i}\right) \\
& =14+\sharp\left(\neg \square^{m-i} \perp\right) \\
& =16+(m-i)
\end{aligned}
$$

Put $\Delta:=\square^{m+1} \perp ;\left\{\varphi_{i}: i<n\right\}$. Then

$$
\begin{aligned}
\sharp\left(\Delta ; \neg\left(\square^{m} \perp \wedge \square^{m-1} \neg \square \perp\right)\right) & =14 m+\sharp\left(\square^{m+1} \perp ; \neg\left(\square^{m} \perp \wedge \square^{m-1} \neg \square \perp\right)\right)-\sharp\left(\neg \square \perp ; \square^{m} \perp\right) \\
& =14 m+((m+2)+(2 m+1))-(3+m) \\
& =16 m
\end{aligned}
$$

Hence, with $n:=16 m$ we get the desired lower bound. For there exist $\Delta$ and $\varphi$ such that $\sharp(\Delta ; \varphi)=n, \Delta \nVdash \varphi$, and the smallest model witnessing this is of size $2^{m+1}-1>2^{n / 16}$.

Now derive the local complexity bounds we take a modified version of the previous example. We have

$$
\square^{m} \perp ;\left\{\square^{j} \varphi_{i}: i, j<m\right\} \nvdash \square^{m} \top \vee \diamond^{m-1} \square \perp
$$


The model constructed earlier serves here as well. Furthermore, a countermodel has at least $2^{m+1}-1$ points, by the same argument. However, the number of subformulae is now $10 m+m(m-1)=m^{2}+9 m=(m+9 / 2)^{2}-(9 / 2)^{2}$. This gives the lower bound, assuming that $n=m^{2}+9 m$.

As we have remarked with the complexity class, these results can be used to derive bounds for the size of models for entire intervals of logics. We will give an example. The logic K.D.alt ${ }_{1}$ has a linear local complexity function, as is easily seen. However, the global complexity function is not even polynomial. For consider the following formulae

$$
\begin{aligned}
\gamma_{n}(p) & :=p \leftrightarrow\left(\square^{n} p \wedge \bigwedge_{0<i<n} \square^{i} \neg p\right) \\
\sharp\left(\gamma_{n}(p)\right) & \left.=7+\sharp\left(p ; \square^{n} p \wedge \bigwedge_{0<i<n} \square^{i} \neg p\right)\right) \\
& =7+n+\sharp\left(\bigwedge_{0<i<n} \square^{i} \neg p\right) \\
& =6+2 n+\sharp\left(\left\{\square^{i} \neg p: 0<i<n\right\}\right) \\
& =6+2 n+\sharp\left(\square^{n-1} \neg p\right) \\
& =7+3 n
\end{aligned}
$$

Now let $q_{i}, i<s$, be the first $n$ prime numbers. Put $\Delta_{n}:=\left\{\gamma_{q_{i}}\left(p_{i}\right): i<n\right\} \cup\left\{\square p_{i} \leftrightarrow\right.$ $\left.\neg \square \neg p_{i}: i<n\right\}$.

Lemma 33 Let L be a modal logic in the interval $\left[\boldsymbol{K}, \boldsymbol{K} . \boldsymbol{D}\right.$. alt $\left._{1}\right]$. Then $\neg \square \perp ; \Delta_{n}$ is globally L-consistent. Moreover, any L-model witnessing this has size $\geq \prod_{i<n} q_{i}$.

Proof. Suppose that $\langle\mathfrak{F}, \beta\rangle$ is a global $L$-model such that $\langle\mathfrak{F}, \beta\rangle \models \neg \square \perp ; \Delta_{n}$. Let $\left\langle w_{i}: i<\omega\right\rangle$ be a sequence of points such that $w_{i} \triangleleft w_{i+1}$ for all $i \in \omega$. Such sequence exists, by the fact that the model satisfies $\neg \square \perp$. Then it follows by choice of the formulae $\gamma_{q_{i}}\left(p_{i}\right)$ that (1) $w_{k} \in \beta\left(q_{i}\right)$ iff $w_{k+q_{i}} \in \beta\left(q_{i}\right),(2) w_{k} \in \beta\left(q_{i}\right)$ iff $w_{k+s} \notin \beta\left(q_{i}\right)$ for all $s<q_{i}$. It follows easily that there is a $k$ such that $w_{k} \vDash p_{i}$ for all $i<n$. Then the smallest number $k^{\prime}>k$ such that $w_{k^{\prime}} \vDash p_{i}$ for all $i<n$ is $k+\prod_{i<n} q_{i}$. This is independent of the chosen sequence. It follows that all $w_{j}$ for $k \leq j<k^{\prime}$ must be distinct. So, there are at least $\prod_{i<n} q_{i}$ many points in this frame. It is easy to construct a K.D.alt ${ }_{1}-$ model with exactly that many points. This shows the global consistency of this set.

Let $g(n)$ be the product of all primes $\leq n$, and let $L(n):=\sharp\left(\Delta_{n} ; \neg \square \perp\right)$. Since the formulae in $\Delta_{n}$ use pairwise distinct variables, we have

$$
\begin{aligned}
L(n) & =\sum_{i<n} \sharp\left(\gamma_{q_{i}}\left(p_{i}\right)\right)+3+7 n \\
& =7 n+\sum_{i<n}\left(8+3 q_{i}\right) \\
& =15 n+3 \sum_{i<n} q_{i}
\end{aligned}
$$


Then $f(n):=g\left(L^{-1}(n)\right)$ is the function measuring the size of the models for these formulae in terms of their length. So, $f$ is a lower bound on the global complexity of K.D.alt ${ }_{1}$. We prove that $f$ grows faster than any polynomial. The proof uses some number theory.

To give a lower bound for $f$, we will establish lower bounds for $L^{-1}$ and $g$. This is sufficient, since $g$ is monotonously increasing. First we will deal with $L^{-1}$. Using the asymptotic formula $q_{n} \sim n \log n$ for the $n$th prime number, where $\log$ is the logarithm to the base $e$, we get that $\sum_{i<n} q_{i}$ is asymptotically equal to $\frac{n^{2}}{2 \log n}$. (See [9].) Namely, taking the integral $\int_{1}^{n / \log n} x \log x d x$ we get $x^{2} \log x / 2-x /\left.2\right|_{1} ^{n / \log n}$, which is asymptotically equal to $\frac{n^{2}}{2 \log n}$. The latter is eventually $<\frac{n^{2}}{c}$, where $c$ is any given positive real number. This allows to conclude that asymptotically $L(n)<n^{2} / c$ for any given $c>0$. Putting $n=L^{-1}(m)$, we get $m<L^{-1}(m)^{2} / c$ from which $\sqrt{m c}<L^{-1}(m)$. Hence, changing $m$ back to $n, L^{-1}(n)>\sqrt{c L(n)}$.

Now $\prod_{p \leq n} p \sim e^{n}$. (Namely, $\vartheta(n)$ is defined to be the sum of the $\log p$, where $p$ is a prime number $\leq n$. It can be shown that $\vartheta(n) \sim n$. For [9] on Page 108, Theorem 5.16 gives $\pi(n) \sim \frac{\vartheta(n)}{\log n}$ and on Page 112, we have $\pi(n) \sim \frac{n}{\log n}$, showing $\frac{\vartheta(n)}{\log n} \sim \frac{n}{\log n}$.) Hence, for any $\varepsilon>0, \prod_{p \leq n} p$ is eventually larger than $e^{(1-\varepsilon) n}$.

Now, given positive real numbers $c$ and $\varepsilon$ we get $f(n)=g\left(L^{-1}(n)\right)>e^{\sqrt{c L(n)}(1-\varepsilon)}$. Since we may choose $c^{2} /(1-\varepsilon)$ rather than $c$ we have that eventually also $f(n)>$ $e^{c \sqrt{n}}$. Now, from Lemma 33 we immediately the following result.

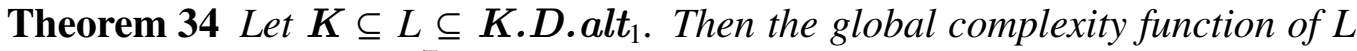
is in the limit at least $2^{c \sqrt{n}}$ for any given $c$.

Let $U$ be a set of worlds in a frame. Let

$$
\begin{array}{ll}
T(U) & :=U \cup\left\{y: x \triangleleft_{j} y, x \in U, j<\kappa\right\} \\
T^{n+1}(U) & :=T\left(T^{n}(U)\right)
\end{array}
$$

$T^{n}(U)$ is the set of all points reachable in at most $n$ steps from a point in $U$. Now say that a frame $\mathfrak{F}$ is of depth $\delta$ if there is a point $x$ such that $T^{\delta}(\{x\})$ is the entire set of worlds from $\mathfrak{F}$.

Definition 35 Let $M$ be a modal logic with the global finite model property. Let $f_{M}$ be the following function. $f_{M}(n)=\max \{\lambda(\Delta ; \varphi): \sharp(\Delta ; \varphi) \leq n\}$, where $\lambda(\Delta ; \varphi)$ is 0 if $\Delta \vdash_{M} \varphi$ and else it is the least number $\delta$ such that there exists a global model $\mathfrak{N}$ of depth $\delta$ such that $\mathfrak{N} \vDash \Delta$ and $\mathfrak{N} \not \models \varphi$.

The local complexity function is defined analogously, where the maximum is taken only over $\lambda(\varnothing ; \varphi)$. 
Theorem 36 Let $d_{g}$ and $d_{\sharp}$ be the global and local depth complexity functions of $\boldsymbol{K}_{\kappa}$. Then the following holds asymptotically, for all $c>0$ :

1. $2^{c \sqrt{n}}<d_{g}(n)<2^{n}$.

2. $d_{\sharp}(n)=n-2$.

The local complexity bound is rather trivial to establish, using the fact that the depth of the models can be chosen to be at most the modal depth of the formulae, which in turn is at most half of the number of subformulae. The upper bound is reached by $\neg \square^{n-1} \perp ; \square^{n} \perp$. This formula has length $n+2$ and needs a model of depth exactly $n$. The global results follow from (the proof of) Theorem 34 and the fact that the depth complexity function never exceeds the global complexity function. It is clear that the theorem does not reveal mucht about transitive logics; here the depth complexity is $\leq 2$. Finally, it is clear that there is an analogue of Theorem 30 with respect to the depth functions.

\section{The Reduction from Global to Local}

Now let $\vdash$ and $r^{\prime}$ be two consequence relations. We call $X$ a reduction function from $r$ to $r^{\prime}$ if

1. $X(\Delta)$ is finite,

2. $\operatorname{var}[X(\Delta)] \subseteq \operatorname{var}[\Delta]$,

3. $X(\Delta) \subseteq \operatorname{Taut}(\vdash)$,

4. $\Delta \vdash \varphi$ iff $\Delta ; X(\Delta ; \varphi) \vdash^{\prime} \varphi$.

In the previous section we have discussed the case where $r$ and $r^{\prime}$ are both global consequence relations. Analogously we can reduce the local consequence relation of $M$ to the local consequence relation of $L$.

Now notice that there is the following connection between local and global consequence relations.

Proposition $37 \Delta \Vdash_{L} \varphi$ iff for some compound modality $\boxplus: ~ \boxplus \Delta \vdash_{L} \varphi$. 
Moreover, we can simplify the choices for $\boxplus$ somewhat. Define for finite $\kappa$ :

$$
\begin{array}{ll}
\square^{0} \varphi & :=\varphi \\
\square^{1} \varphi & :=\bigwedge_{j<k} \varphi \\
\square^{k+1} \varphi & :=\square^{1} \square^{k} \varphi \\
\square^{\leq k} \varphi & :=\bigwedge_{i \leq k} \square^{i} \varphi
\end{array}
$$

Definition 38 Assume that $\kappa$ is finite. Let $f$ be a function from finite sets of formulae to $\omega . \quad f$ is a global-to-local reduction function or gl-reduction function for L iffor all finite sets $\Delta$ and $\varphi$

$$
\Delta \Vdash_{L} \varphi \quad \Leftrightarrow \quad \square^{\leq k} \Delta \vdash_{L} \varphi
$$

where $k:=f(\Delta ; \varphi)$.

The following theorem is easy to show.

Proposition 39 Suppose that $L$ is locally decidable and that there is a computable gl-reduction function for $L$. Then $L$ is globally decidable.

To put it negatively: if a logic is locally decidable but globally undecidable, then no computable gl-reduction function for $L$ exists. [15] has proved the existence of such logics. A logic is weakly transitive if it has a theorem of the form $\square^{k} p \rightarrow$ $\square^{k+1} p$. Clearly, weakly transitive logics have computable reduction functions: simply put $f(\Delta):=k$. Furthermore, observe the following.

Proposition 40 Suppose that $M$ has a (computable) global reduction function to $L$ and that $L$ has a (computable) gl-reduction function. Then $M$ has a (computable) gl-reduction function.

Proof. Let $X$ be a reduction function from $M$ to $L$, and $f$ a gl-reduction function for $L$. Then

$$
\begin{aligned}
\Delta \Vdash_{M} \varphi & \Rightarrow \Delta ; X(\Delta ; \varphi) \Vdash_{L} \varphi \\
& \Rightarrow \square^{\leq k} \Delta ; \square^{\leq k} X(\Delta ; \varphi) \vdash_{L} \varphi \\
& \Rightarrow \square^{\leq k} \Delta ; \square^{\leq k} X(\Delta ; \varphi) \vdash_{M} \varphi \\
& \Rightarrow \square^{\leq k} \Delta \vdash_{M} \varphi
\end{aligned}
$$

where $k:=f(\Delta ; \varphi ; X(\Delta ; \varphi))$. Hence put $g(\Delta):=f(\Delta ; X(\Delta))$. This is a gl-reduction function for $M$. If both $f$ and $X$ are computable, then so is $g$.

A logic $M$ is called weakly transitive if there exists a compound modality $\square$ such that $\otimes p \rightarrow p, \otimes p \rightarrow \otimes \otimes p, \otimes p \rightarrow \square_{j} p$ and $\otimes p \rightarrow \square_{j} \otimes p$ are theorems 
of $M$ for all modal operators $\square_{j}$. We call $\otimes$ a master modality. If $M$ is weakly transitive with master modality $\square$ then

$$
\Delta \Vdash_{M} \varphi \quad \Leftrightarrow \quad \nabla \Delta \vdash_{M} \varphi
$$

Moreover, $\sharp(\otimes \Delta) \leq c \cdot \sharp(\Delta)$ for some constant $c$. Hence, the global derivability is linearly recodable as a local derivability problem. So we have the

Proposition 41 Suppose that $M$ is weakly transitive. Then there exists a linear gl-reduction function for $M$.

It follows that in the weakly transitive case the local and the global complexity coincide. For we generally have

$$
\Delta \vdash_{M} \varphi \quad \Leftrightarrow \quad \Vdash_{M} \bigwedge \Delta \rightarrow \varphi
$$

Moreover, $\sharp(\wedge \Delta \rightarrow \varphi) \leq 2+2 \sharp(\Delta ; \varphi)$. So, the local derivability problem is linearly recodable into a global derivability problem.

We will discuss a special case to solve, and that is the gl-reduction of $\mathbf{K}_{\kappa}$. In [7] the following is proved. (We reproduce the proof here.)

Theorem 42 Let $\Delta$ be a finite set of formulae and $\varphi$ a formula. Put $k:=2^{\sharp(\Delta)+\sharp(\varphi)}$. Then

$$
\Delta \Vdash_{\mathbf{K}_{\kappa}} \varphi \quad \Leftrightarrow \quad \square^{\leq k} \Delta \vdash_{\mathbf{K}_{\kappa}} \varphi
$$

Proof. Assume $\square^{\leq k} \varphi \nvdash_{\mathbf{K}_{k}} \psi$. Then there exists a finite model $\left\langle\mathfrak{F}, \beta, w_{0}\right\rangle \vDash$ $\square^{\leq k} \varphi ; \neg \psi$. Moreover, we may assume that $\mathfrak{F}$ is cycle-free, and that between any pair of points there exists at most one path. Let $\Delta:=s f(\varphi) \cup s f(\psi)$ and put $S(y)=\{\chi \in \Delta:\langle\mathfrak{F}, \beta, y\rangle \vDash \chi\}$. Let $G$ be the set of all $y$ in $F$ such that along any path from $w_{0}$ to $y$ there are no two distinct points $v$ and $w$ such that $S(v)=S(w)$. Then any path from $w_{0}$ to $y \in G$ has length $\leq k$, because there are at most $k$ subsets of $\Delta$. Now define $\triangleleft_{j}$ on $G$ as follows. $y \triangleleft_{j} z$ iff (1) $y \triangleleft_{j} z$ or (2) for some $u \notin G$ we have $y \triangleleft_{j} u$ and $S(z)=S(u)$. Put $\gamma(p):=\beta(p) \cap G$. We will now show that for every $y \in G$ and $\chi \in \Delta$

$$
\langle\mathfrak{G}, \gamma, y\rangle \vDash \chi \quad \Leftrightarrow \quad\langle\mathfrak{F}, \beta, y\rangle \vDash \chi .
$$

This is true for variables by construction. The steps for negation and conjunction are clear. Now let $\chi=\diamond_{j} \delta$. If $\langle\mathfrak{F}, \beta, y\rangle \vDash \diamond_{j} \delta$ then for some $z$ such that $y \triangleleft_{j} z$ we have $\langle\mathfrak{F}, \beta, z\rangle \vDash \delta$. There are two cases. Case 1. $z \in G$. Then by induction 
hypothesis, $\langle\mathfrak{G}, \gamma, z\rangle \vDash \delta$. From this we conclude $\langle\mathfrak{G}, \gamma, y\rangle \vDash \diamond_{j} \delta$, since $y \boldsymbol{\triangleleft}_{j} z$. Case 2. $z \notin G$. Then there is a $u \in G$ such that $S(u)=S(z)$. Therefore, by construction of $\mathfrak{G}, y \boldsymbol{\triangleleft}_{j} u$. Furthermore, $\langle\mathfrak{F}, \beta, u\rangle \vDash \delta$ by definition of $S(-)$. So, $\langle\mathfrak{G}, \gamma, u\rangle \vDash \delta$ by induction hypothesis. From this follows $\langle\mathfrak{G}, \gamma, y\rangle \vDash \diamond_{j} \delta$, since $y \varangle_{j} u$. This exhausts the two cases. Now suppose $\langle\mathfrak{G}, \gamma, y\rangle \vDash \diamond_{j} \delta$. Then $\langle\mathfrak{G}, \gamma, z\rangle \vDash \delta$ for some $z$ such that $y \triangleleft_{j} z$. By induction hypothesis, $\langle\mathfrak{F}, \beta, z\rangle \vDash \delta$. If $y \triangleleft_{j} z$, then also $\langle\mathfrak{F}, \beta, y\rangle \vDash \diamond_{j} \delta$. If, however, $y 丸_{j} z$, then there is a $u$ such that $y \triangleleft_{j} u$ and $S(u)=S(z)$. By definition of $S(-),\langle\mathfrak{F}, \beta, u\rangle \vDash \delta$, from which $\langle\mathfrak{F}, \beta, y\rangle \vDash \diamond_{j} \delta$ as well. Now since from $w_{0}$ there is always a path of length $\leq 2^{k}$ to any point $y \in G$, we have $\langle\mathfrak{F}, \beta, y\rangle \vDash \varphi$ for all $y \in G$, and so $\langle\mathfrak{G}, \beta, y\rangle \vDash \varphi$ for all $y$. Consequently, $\left\langle\mathfrak{G}, \beta, w_{0}\right\rangle \vDash \square^{\omega} \varphi ; \neg \psi$, as required.

\section{Interpolation and Beth Theorems}

Definition 43 A modal logic L has local interpolation if for every pair $\varphi$ and $\psi$ of formulae with $\varphi \vdash_{L} \psi$ there is a $\chi$ such that $\operatorname{var}(\chi) \subseteq \operatorname{var}(\varphi) \cap \operatorname{var}(\psi)$ and $\varphi \vdash_{L} \chi$ as well as $\chi \vdash_{L} \psi$. L has global interpolation if for every pair $\varphi, \psi$ of formulae with $\varphi \Vdash_{L} \psi$ there is a $\chi$ such that $\operatorname{var}(\chi) \subseteq \operatorname{var}(\varphi) \cap \operatorname{var}(\psi)$ and $\varphi \Vdash_{L} \chi$ as well as $\chi \Vdash_{L} \psi$.

Since we have a deduction theorem for local deducibility, we can reformulate local interpolation in such a way that it depends only on the set of theorems. $L$ has the Craig Interpolation Property if whenever $\varphi \rightarrow \psi \in L$ there exists a $\chi$ which is based on the common variables of $\varphi$ and $\psi$ such that $\varphi \rightarrow \chi ; \chi \rightarrow \psi \in L$. A logic has the Craig Interpolation Property iff it has local interpolation.

Proposition 44 If L has local interpolation it also has global interpolation.

Proof. Suppose that $L$ has local interpolation. Let $\varphi \Vdash_{L} \psi$. Then for some compound modality $\otimes$ we have $\nabla \varphi \vdash_{L} \psi$. Whence by local interpolation there is a $\chi$ with $\operatorname{var}(\chi) \subseteq \operatorname{var}(\varphi) \cap \operatorname{var}(\psi)$ such that $\otimes \varphi \vdash_{L} \chi$ and $\chi \vdash_{L} \psi$. Hence $\varphi \Vdash_{L} \chi$ as well as $\chi \Vdash_{L} \psi$.

The converse implication does not hold, as has been shown in [12]. Interpolation is closely connected with the so-called Beth property. It says, in intuitive terms, that if we have defined $p$ implicitly, then there also is an explicit definition of $p$. An explicit definition is a statement of the form $\chi \leftrightarrow p$ where $p \notin \operatorname{var}(\chi)$. An implicit definition is a formula $\psi(p, \vec{q})$, such that the value of $p$ in a model is uniquely 
defined by the values of the variables $\vec{q}$. The latter can be reformulated syntactically. Given a consequence relation $\vdash$, we say that $\varphi(p, \vec{q})$ implicitly defines $p$ (in $\vdash$ ) if $\varphi(p, \vec{q}) ; \varphi(r, \vec{q}) \vdash p \leftrightarrow r$. Given $L$, we may choose $\vdash$ to be either $\vdash_{L}$ or $\vdash_{L}$. This gives rise to the notions of local and global implicit definitions.

Definition 45 L is said to have the local Beth Property if the following holds. Suppose $\varphi(p, \vec{q})$ is a formula and

$$
\varphi(p, \vec{q}) ; \varphi(r, \vec{q}) \vdash_{L} p \leftrightarrow r .
$$

Then there exists a formula $\chi(\vec{q})$ not containing $p$ as a variable such that

$$
\varphi(p, \vec{q}) \vdash_{L} p \leftrightarrow \chi(\vec{q}) .
$$

Analogously, the global Beth property is defined by replacing $\vdash_{L}$ by $\vdash_{L}$.

The lack of the deduction theorem for the global consequence makes the global Beth property somewhat more difficult to handle than the local equivalent. For the local Beth property we can actually prove that it is equivalent to the Craig Interpolation Property. The following two results were shown in [12].

Theorem 46 (Maksimova) Let L be a modal logic. Then L has local interpolation iff it has the local Beth property.

Theorem 47 (Maksimova) A modal logic with local interpolation also has the global Beth-property.

Proof. Assume that $\varphi(p, \vec{q}) ; \varphi(r, \vec{q}) \Vdash_{L} p \leftrightarrow r$. Then for some compound modality $\triangle$ we have

$$
\bigotimes \varphi(p, \vec{q}) ; \bigotimes \varphi(r, \vec{q}) \vdash_{L} p \leftrightarrow r .
$$

This can now be rearranged to

$$
\bigotimes \varphi(p, \vec{q}) ; p \vdash_{L} \otimes \varphi(r, \vec{q}) \rightarrow r .
$$

We get an interpolant $\chi(\vec{q})$ and so we have $\otimes \varphi(p, \vec{q}) ; p \vdash_{L} \chi(\vec{q})$, from which $\nabla \varphi(p, \vec{q}) \vdash_{L} p \rightarrow \chi(\vec{q})$. So $\varphi(p, \vec{q}) \vdash_{L} p \rightarrow \chi(\vec{q})$. And we have $\chi(\vec{q}) \vdash_{L} \otimes \varphi(r, \vec{q}) \rightarrow$ $r$ from which we get $\otimes \varphi(r, \vec{q}) \vdash_{L} \chi(\vec{q}) \rightarrow r$, and so $\varphi(r, \vec{q}) \vdash_{L} \chi(\vec{q}) \rightarrow r$. Replacing $r$ by $p$ we get the desired result.

The picture obtained thus far is the following. 


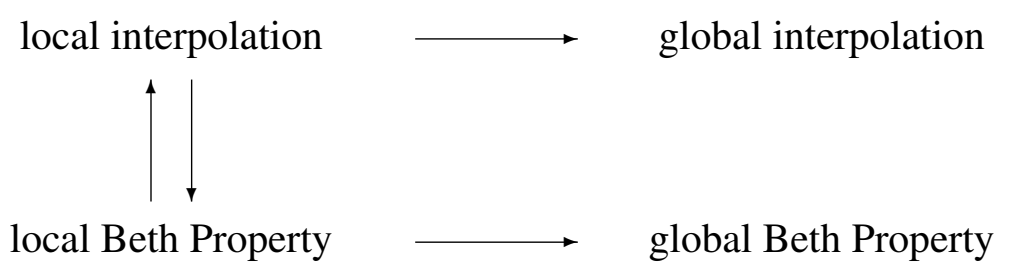

It can be shown that there exist logics without global interpolation while having the global Beth Property and that there exist logics with global interpolation without the global Beth Property. An example of the first kind is the logic G.3. (See [11].)

Definition 48 Let L be a modal logic. L is locally Halldén-complete if whenever $\varphi \vdash_{L} \psi$ and $\operatorname{var}(\varphi) \cap \operatorname{var}(\psi)=\varnothing$ we have $\varphi \vdash_{L} \perp$ or $\vdash_{L} \psi$. L is globally Halldén-complete if whenever $\varphi \Vdash_{L} \psi$ and $\operatorname{var}(\varphi) \cap \operatorname{var}(\psi)=\varnothing$ we have $\varphi \Vdash_{L} \perp$ or $\Vdash_{L} \psi$.

Global Halldén-completeness is called the Pseudo Relevance Property in [13]. In the literature, a logic $L$ is called Halldén-complete if for $\varphi$ and $\psi$ disjoint in variables, if $\varphi \vee \psi \in L$ then also $\varphi \in L$ or $\psi \in L$. Clearly, this latter notion of Halldén-completeness coincides with local Halldén-completeness. This follows from the deduction theorem, since $\varphi \vdash_{L} \psi$ is equivalent to $\vdash_{L} \neg \varphi \vee \psi$. If a logic is locally Halldén-complete it is also globally Halldén-complete. For if $\varphi \Vdash_{L} \psi$ then $\boxplus \varphi \vdash_{L} \psi$. If $\varphi$ and $\psi$ have no variable in common, this holds for $\boxplus \varphi$ and $\psi$ as well. Hence, $\varphi \vdash_{L} \perp$ or else $\vdash_{L} \psi$, from which we get $\varphi \Vdash_{L} \perp$ or $\Vdash_{L} \psi$. Further, if $L$ logic is locally Halldén-complete then any constant formula must be equivalent to $\perp$ or $T$. This is the case, for example, when all modal operators are reflexive.

Proposition 49 Suppose that $L$ is a logic such that every constant formula is locally equivalent to $\perp$ or $\mathrm{T}$. Then if L has local (global) interpolation, it is locally (globally) Halldén-complete.

For a proof note that if $\varphi \vdash_{L} \psi$ with $\varphi$ and $\psi$ disjoint in variables, then there exists a constant formula $\chi$ such that $\varphi \vdash_{L} \chi$ and $\psi \vdash_{L} \psi$. By assumption on $L, \chi$ is equivalent to $T$ or $\perp$. If the first holds then $T \vdash_{L} \psi$ and if the second holds then $\varphi \vdash_{L} \psi$. Similarly for the global case.

Finally, we will establish some criteria for interpolation. Assume that we have $\operatorname{logics} L$ and $L^{\prime}$ with $L \subseteq L$ and global reduction sets for $L^{\prime}$ with respect to $L$. Let us say that the reduction sets split if there exists a reduction function $X$ such that $X(\varphi ; \psi)=X(\varphi \rightarrow \psi)=X(\varphi) \cup X(\psi)$. 
Theorem 50 Suppose that $L^{\prime}$ can be globally reduced to L with splitting reduction sets. Then $L^{\prime}$ has local (global) interpolation if L has local (global) interpolation. Moreover, $L^{\prime}$ is locally (globally) Halldén-complete if $L$ is.

Proof. Assume $\varphi \vdash_{L^{\prime}} \psi$. Using the deduction theorem we get $\vdash_{L^{\prime}} \varphi \rightarrow \psi$. Then $\Vdash_{L^{\prime}} \varphi \rightarrow \psi$. By global reduction we get $X(\varphi \rightarrow \psi) \Vdash_{L} \varphi \rightarrow \psi$ and so for some compound modality $\otimes$

$$
\boxplus X(\varphi \rightarrow \psi) \vdash_{L} \varphi \rightarrow \psi
$$

This is the same as

$$
\boxplus X(\varphi) ; \boxplus X(\psi) \vdash_{L} \varphi \rightarrow \psi,
$$

by the assumption that the reduction sets split. We can rearrange this into

$$
\boxplus X(\varphi) ; \varphi \vdash_{L} \boxplus X(\psi) \rightarrow \psi
$$

(We allow ourselves to write $\boxplus X(\psi)$ in place of $\bigwedge \boxplus X(\psi)$.) By assumption on $X, \operatorname{var}[X(\varphi)] \subseteq \operatorname{var}(\varphi)$ and $\operatorname{var}[X(\psi)] \subseteq \operatorname{var}(\psi)$. By local interpolation for $L$ we obtain a $\tau$ in the common variables of $\varphi$ and $\psi$ such that

$$
\varphi ; \boxplus X(\varphi) \vdash_{L} \tau \vdash_{L} \boxplus X(\psi) \rightarrow \psi
$$

From this follows that $\varphi \vdash_{L^{\prime}} \tau \vdash_{L^{\prime}} \psi$, by the fact that the reduction sets only contain instances of theorems. Moreover, $\operatorname{var}(\tau) \subseteq \operatorname{var}(\varphi) \cap \operatorname{var}(\psi)$. Pushing up global interpolation works essentially in the same way. Now, for Halldén-completeness, assume that $\varphi \vdash_{L^{\prime}} \psi$ for $\varphi$ and $\psi$ disjoint in variables. Then

$$
\varphi ; \boxplus X(\varphi) \vdash_{L} \boxplus X(\psi) \rightarrow \psi
$$

The left hand side is disjoint in variables from the right hand side, and so either the left hand side is inconsistent or the right hand side is a theorem. In the first case, $\varphi \vdash_{L^{\prime}} \perp$. In the second case $\vdash_{L^{\prime}} \psi$, as required. The proof for global Halldéncompleteness is analogous.

We conclude from that the following theorem.

Corollary 51 The monomodal logics K.alt, K4, K.B, K.T, K.BT, S4, S5, $G$ and $G r z$ have local interpolation. Moreover, $K . T, K . B T, S 4, S 5$ and $G r z$ are Halldén-complete.

This is so since the reduction functions given earlier split, as an easy inspection reveals. The second claim follows from Proposition 49. The following has been observed first in [14] 
Corollary 52 (Rautenberg) Let L have local (global) interpolation and let $\Psi$ be a set of constant formulae. Then $L \oplus \Psi$ has local (global) interpolation.

By considerations analogous to Theorem $13, X_{\Psi}(\Delta ; \psi):=\Psi$ is a global reduction function. Obviously, $X$ splits. An analogous theorem holds for local and global Halldén-completeness; however it is of no use. For if a logic is Halldén-complete, any constant formula is equivalent to either $\perp$ or $T$.

We close with a remark on interpolation. Say that $L$ has constructible local interpolants if for given $\varphi$ and $\psi$ such that $\varphi \vdash_{L} \psi$ we can construct a local interpolant $\chi$. It can be shown that if $M$ can be locally constructively reduced to $L$ and $L$ has constructible local interpolants, then this holds for $M$ as well. Similarly for global interpolation. ${ }^{1}$

\section{Conclusion}

We have introduced the notion of a reduction function and shown how to use reduction functions to prove certain standard and some new results about decidability, finite model property, interpolation etc. of logical systems. All methods are constructive: the reduction functions are shown to be constructible if the logic is decidable. Moreover, if we are given reduction function, we assume that the base logic $L$ has the finite model property and construct an $M$-model on the basis of a suitably defined $L$-model. The only handicap of the method is the fact that we use a global reduction function instead of a local one, even though we show also that the global consequence relation is reducible in the same way to the local reducibility. Yet, the bounds obtained in this way for the time and space complexity are sometimes far off the mark. However, our results are - so we think only the beginning. It is conceivable that they can be improved to establish the bounds known from the literature.

\section{Appendix: Subframe Logics containing K4}

In this appendix, all frames and logics are assumed to be transitive. A subframe $\mathfrak{G}$ is cofinal in $\mathfrak{F}$ if every point in the subframe generated by $\mathfrak{G}$ in $\mathfrak{F}$ is covered by a point of $\mathfrak{G}$. That means, if $x \in G$ and $x \triangleleft y$ then either $y \in G$ or else $y \triangleleft z$ for some $z \in G$. A logic is called a cofinal subframe logic if its class of frames is closed

\footnotetext{
${ }^{1}$ I owe this observation to Oliver Kutz.
} 
under taking cofinal subframes. Obviously, a subframe logic is a cofinal subframe logic; the converse need not hold, e. g. S4.2. Examples of subframe logics are S4, S5, G, Grz, K4.3 and many more.

We will prove in this appendix the following theorem, proved in [3] for subframe logics and in [16] for cofinal subframe logics.

Theorem 53 (Fine, Zakharyaschev) Every cofinal subframe logic containing $K_{4}$ has the finite model property.

For subframe logics this is due to Kit Fine; the generalization to cofinal subframe logics (as well as Fine's result independently) has been obtained by Michael Zakharyaschev. Before we enter the proof let us introduce some useful terminology and draw important consequences. If $L$ is a logic and $\mathfrak{F}$ rooted and finite then denote by $L /_{\mathrm{F}} \mathfrak{F}$ the smallest subframe logic containing $L$ not having $\mathfrak{F}$ as a frame, and call $L /_{\mathrm{F}} \mathfrak{F}$ the Fine-splitting of $L$ by $\mathfrak{F}$. It turns out that $L / \mathrm{F} \mathfrak{F}=L \oplus C_{\mathfrak{F}}$ where $C_{\mathfrak{F}}=\square^{+} S F(\mathfrak{F}) . \rightarrow . \neg p_{o}$ with $o$ a root

$$
\begin{aligned}
S F(\mathfrak{F}):= & \wedge\left\langle p_{x} \rightarrow \neg p_{y}: x \neq y\right\rangle \\
& \wedge \bigwedge\left\langle p_{x} \rightarrow \diamond p_{y}: x \triangleleft y\right\rangle \\
& \wedge \bigwedge\left\langle p_{x} \rightarrow \neg \nabla p_{y}: x \not y\right\rangle
\end{aligned}
$$

Any subframe logic $L$ is a Fine-splitting $\mathbf{K 4} /{ }_{\mathrm{F}} G$ with $G=\{\mathfrak{F}: \mathfrak{F} \notin \operatorname{Fr}(L), \mathfrak{G}$ rooted $\}$. Analogously, a Zakharyaschev-splitting of $L$ by $\mathfrak{F}$ is the least cofinal subframe logic containing $L$ for which $\mathfrak{F}$ is not a frame. It is axiomatizable by $L \oplus \square^{+} C S F(\mathfrak{F}) . \rightarrow$ $\neg p_{o}$. We write $L / \mathrm{z} \mathfrak{F}$.

$$
\begin{aligned}
C S F(\mathfrak{F}):= & \wedge\left\langle p_{x} \rightarrow \neg p_{y}: x \neq y\right\rangle \\
& \wedge \bigwedge\left\langle p_{x} \rightarrow \diamond p_{y}: x \triangleleft y\right\rangle \\
& \wedge \bigwedge\left\langle p_{x} \rightarrow \neg \nabla p_{y}: x \not y\right\rangle \\
& \wedge \square \diamond \bigvee\left\langle p_{x}: x \in f\right\rangle
\end{aligned}
$$

Put $\mathfrak{F} \prec_{F} \mathfrak{G}$ if $\mathfrak{G}$ is a p-morphic image of a subframe of $\mathfrak{F}$, and $\mathfrak{F} \prec_{Z} \mathfrak{G}$ if $\mathfrak{G}$ is a p-morphic image of a cofinal subframe of $\mathfrak{F}$. Then $\prec_{Z} \subseteq<_{F}$. If $\mathfrak{F}$ is a K4-frame and $x, y \in f$ then $y$ is called a weak successor of $x$, in symbols $x \unlhd y$, if $x \triangleleft y$ or $x=y . y$ is a strong successor of $x$, in symbols $x \vec{\triangleleft} y$, if $x \triangleleft x$ but $y \nless x$. The cluster $C(x)$ of $x$ is the set of weak successors which are not strong, that is the set of $y$ such that $x \unlhd y \unlhd x$. The depth of a point $x, d(x)$, is the maximum number $n$ such that there is a sequence $x=x_{0} \vec{\triangleleft} x_{1} \vec{\triangleleft} \ldots \vec{\triangleleft} x_{n-2} \vec{\triangleleft} x_{n}$. In the sequel we fix a formula $\varphi$, and let $S:=s f(\varphi)$. Let $\mathfrak{M}=\langle\mathfrak{F}, \beta\rangle$ be a model. We make the crucial assumption from now on that the $\beta(p), p \in \operatorname{var}(\varphi)$, generate the algebra of sets of 
$\mathfrak{F}$. We say in that case that the model is $\varphi$-refined. In a model, the characteristic set of a point $x, X(x)$, is the set of all formulae of $S=s f(\varphi)$ true at $x$. The atom or characteristic formula is

$$
\operatorname{At}(x):=\bigwedge_{\psi \in X(x)} \psi \wedge \bigwedge_{\psi \notin X(x)} \neg \psi
$$

The set of all conjunctions of this type, i. e. the set of all characteristic formulae, is denoted by $A(\varphi)$ or simply $A$. Call $x \in F$ maximal (with respect to $\varphi$ ) in $\mathfrak{M}$ if no strong successor of $x$ has the same characteristic set (or formula) as $x$. The subframe of all maximal points of $\mathfrak{M}=\langle\mathfrak{F}, \beta\rangle$ is denoted by $\mathfrak{F}^{\mu}$. In a finite model, every point $x$ has a weak successor which is maximal for the atom of $x$. This weak successor is denoted by $x^{\mu}$. Then $x=x^{\mu}$ iff $x$ is maximal (by $\varphi-$ refinement). Hence, $\mathfrak{F}^{\mu}$ is actually cofinal in $\mathfrak{F}$. It is useful to observe that if $x \triangleleft y^{\mu}$ and $x=\diamond A t\left(y^{\mu}\right)$ then there is a weak successor $x^{\mu}$ such that $x^{\mu} \triangleleft y^{\mu}$. We now have the following

Proposition 54 Let $\mathfrak{F}^{\mu} \subseteq \mathfrak{G} \subseteq \mathfrak{F}$ and $x \in G$. Then for all $\varphi \in S$ :

$$
\langle\mathfrak{F}, \beta, x\rangle \vDash \varphi \quad \Leftrightarrow \quad\langle\mathfrak{G}, \beta, x\rangle \vDash \varphi
$$

The proof of this theorem is straightforward. As a consequence, there is no distinction whether we choose maximal points in $\mathfrak{F}$ or in $\mathfrak{G}$. They will be the same set. And so $x^{\mu}$ does not depend on $\mathfrak{G}$ as long as $G \supseteq F^{\mu}$. Let $\mathfrak{R}$ be the refinement of the frame $\mathfrak{F}^{\mu}$, where the internal sets are those generated by $\beta\left(p_{i}\right), p_{i} \in \operatorname{var}(\varphi)$. (That is to say, we consider the general frame based on $\mathfrak{F}^{\mu}$, in which the algebra of sets is generated by the values of the variables $p_{i}$.) Write $\beta$ for the valuation induced on $\Re$. Let $d^{\mu}(x)$ be the depth of $x$ in $\Re$.

Fact 55 For all $x \in G, d^{\mu}(x)$ is the maximum number $n$ such that there is a chain $\left\langle x_{i}: i \in n\right\rangle$ with $x_{0}=x, x_{i} \vec{\triangleleft} x_{i+1}$, and $x_{i+1} \vDash \neg A t\left(x_{i}\right) \wedge \neg \vee A t\left(x_{i}\right), i \in n-1$. By consequence, $d^{\mu}(x) \leq 2^{\sharp S}$.

Proof. This is seen by first noting that if there is a chain $\left\langle x_{i}: i \in n\right\rangle$ such that $x_{i+1} \vDash$ $\neg A t\left(x_{i}\right) \wedge \neg \checkmark A t\left(x_{i}\right)$, then $x_{i+1} \Varangle x_{i}$, and, starting with $x_{n-1}$, one can successively replace the $x_{i}$ by a maximal weak successor $x_{i}^{\mu}$ so that $x_{i}^{\mu} \vec{\forall} x_{i+1}^{\mu}$. Conversely, if there is a chain $\left\langle x_{i}: i \in n\right\rangle$ of maximal points such that $x_{i} \vec{\triangleleft} x_{i+1}$ then $x_{i+1} \vDash$ $\neg A t\left(x_{i}\right) \wedge \neg \checkmark A t\left(x_{i}\right)$. So there is a chain of points with $x_{i+1} \vDash \neg A t\left(x_{i}\right) \wedge \neg \vee A t\left(x_{i}\right)$ iff there is a chain of maximal points of the same length satisfying $x_{i} \vec{\triangleleft} x_{i+1}$ iff $d^{\mu}\left(x_{0}\right) \geq n$. 
For $x \in F$ define

$$
\begin{array}{ll}
\operatorname{suc}^{+}(x) & :=\left\{y \in F^{\mu}: x \vec{\triangleleft} y\right\}, \\
\operatorname{cl}(x) & :=\left\{y \in F^{\mu}: x \triangleleft y \triangleleft x\right\}, \\
C L(x) & :=\{\operatorname{At}(y): y \in \operatorname{cl}(x), y \text { maximal }\}
\end{array}
$$

By induction on $d^{\mu}(x)$ we will now define formulae $\varepsilon_{x}, \lambda_{x}$. The formulae $\varepsilon_{x}$ will encode the structure of the refined submodel of maximal points. The formulas $\lambda_{x}$ define the layers of that model, that is, the set of all points $y$ in the submodel of maximal points with $d^{\mu}(y)<d^{\mu}(x)$. The induction starts with $d^{\mu}(x)=-1$, where there is nothing to do, except to let $\lambda_{-1}:=\perp$. Now let $d^{\mu}(x):=d+1$ with $d \geq-1$. (We write $\diamond^{+} \varphi$ for $\varphi \vee \diamond \varphi$ and $\square^{+} \varphi$ for $\varphi \wedge \square \varphi$.)

$$
\begin{aligned}
& \lambda_{x} \quad:=\zeta_{d} \\
& \alpha_{x} \quad:=\operatorname{At}(x) \wedge \neg \lambda_{x} \wedge \square\left(\lambda_{x} \rightarrow \neg \vee^{+} \operatorname{At}(x)\right)
\end{aligned}
$$

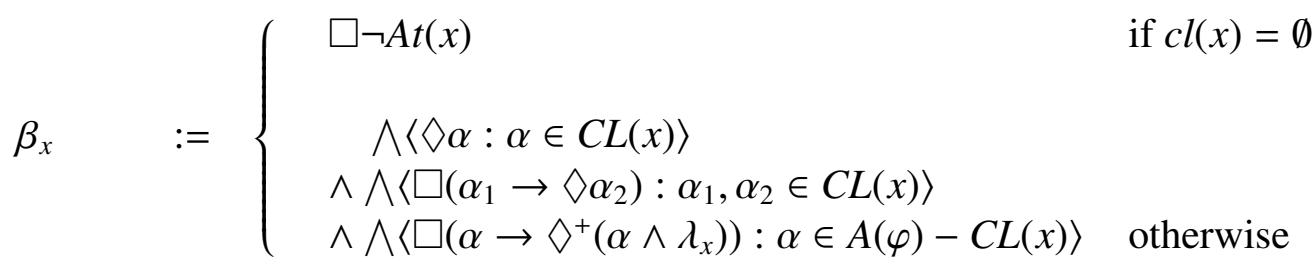

$$
\begin{aligned}
& \gamma_{x} \quad:=\left\{\begin{array}{l}
\bigwedge\left\langle\diamond \varepsilon_{y} \wedge \square\left(\diamond^{+} A t(x) \rightarrow \diamond \varepsilon_{y}\right): y \in \operatorname{suc}^{+}(x)\right\rangle \\
\wedge \bigwedge\left\langle\square \neg \varepsilon_{y}: y \notin \operatorname{suc} c^{+}(x), d^{\mu}(y) \leq d\right\rangle \\
\wedge \bigwedge\left\langle\square\left(\square \neg \varepsilon_{y} \rightarrow \bigvee_{\alpha \in A(\varphi)-C L(x)} \diamond^{+}\left(\alpha \wedge \lambda_{x}\right)\right): y \in \operatorname{suc}^{+}(x)\right\rangle
\end{array}\right. \\
& \varepsilon_{x} \quad:=\alpha_{x} \wedge \beta_{x} \wedge \gamma_{x} \\
& \zeta_{d+1}:=\quad:=\quad \zeta_{d} \vee \bigvee\left\langle\varepsilon_{x}: d^{\mu}(x)=d+1\right\rangle
\end{aligned}
$$

Define $S U C^{+}(x):=\left\{\varepsilon_{y}: y \in \operatorname{suc}^{+}(x)\right\}$. Then if $\equiv$ denotes equivalence in $\mathbf{K}$ it is calculated that $\varepsilon_{x} \equiv \varepsilon_{y}$ iff either $(\alpha) S U C^{+}(x)=S U C^{+}(y)$, or $(\beta) C L(x)=C L(y)$ and $A t(x)=A t(y)$. Define the frame $\mathfrak{d e s}=\langle\delta, \triangleleft\rangle$ with $\delta=\left\{\varepsilon_{x} \equiv: x \in F\right\}$ and $\varepsilon_{x} \triangleleft \varepsilon_{y}$ iff either $\varepsilon_{y} \in S U C^{+}(x)$ or $S U C^{+}(x)=S U C^{+}(y), C L(x)=C L(y)$ and $A t(y) \in C L(x)$. (Henceforth we will not distinguish between $\varepsilon_{x}$ and its equivalence class $\varepsilon_{x} \equiv$.) Our aim is to show that $\mathfrak{d e s}$ is nothing but $\mathfrak{R}$, and that the natural valuation on $\mathfrak{d e s}$ is $\beta$. The first lemma shows that the definition of the $\varepsilon_{s}$ is sound for the maximal points: 
Lemma 56 Let $\mathfrak{F}^{\mu} \subseteq \mathfrak{G} \subseteq \mathfrak{F}$ and $x \in \mathfrak{G}^{\mu}$. Then $x \in f^{\mu}$ and $\langle\mathfrak{G}, \beta, s\rangle \vDash \varepsilon_{x}$.

Proof. If $x \in \mathfrak{G}^{\mu}$, then its maximal successor $x^{\mu}$ is in $\mathfrak{G}$, since $\mathfrak{G} \supseteq \mathfrak{F}^{\mu}$. Hence, $x=x^{\mu}$, since $x$ is maximal in $\mathfrak{G}$ and it follows that $x$ is maximal in $\mathfrak{F}$ as well. By induction on $d:=d^{\mu}(x)$ we show

$$
\langle\mathfrak{G}, \beta, x\rangle \vDash \varepsilon_{x} ; \quad \text { moreover, if }\langle\mathfrak{G}, \beta, x\rangle \vDash \lambda_{d} \text { then } d^{\mu}(x) \leq d .
$$

To begin with $d^{\mu}(x)=-1$, there is nothing to show. Thus let $d^{\mu}(x)=d+1$. The proof is broken down into four parts:

(i) $x \vDash \alpha_{x}$

This is so because $y \vDash \lambda_{x}$ implies $y^{\mu} \vDash \lambda_{x}\left(=\zeta_{d}\right)$ by Proposition 54, from which $d^{\mu}\left(y^{\mu}\right) \leq d$. But no maximal successor of $x$ of depth $\leq d$ can satisfy $\operatorname{At}(x)$ or $\diamond A t(x)$.

(ii) $x \models \beta_{x}$

The case $C L(x)=\emptyset$ is straightforward. Let therefore $C L(x) \neq \emptyset$. By definition of $C L(x)$ and the fact that $G \supseteq F^{\mu}$ we get $x \vDash \diamond \alpha_{1} ; \square\left(\alpha_{1} \rightarrow \diamond \alpha_{2}\right)$ for all $\alpha_{1}, \alpha_{2} \in$ $C L(x)$. Also $x \vDash \square\left(\widehat{\alpha} . \rightarrow \diamond^{+}\left(\widehat{\alpha} \wedge \lambda_{x}\right)\right)$ for $\widehat{\alpha} \notin C L(x)$, for if for a successor $y$ : $y \vDash \widehat{\alpha}$, then $y^{\mu} \vDash \widehat{\alpha}$ and by induction hypothesis and the fact that $d^{\mu}\left(y^{\mu}\right) \leq d$, $y^{\mu} \vDash \lambda_{x}$. Thus if $y=y^{\mu}$ we have $y \vDash \lambda_{x}$ and if $y \triangleleft y^{\mu}$ we have $y^{\mu} \vDash \diamond\left(\widehat{\alpha} \wedge \lambda_{x}\right)$.

(iii) $x \vDash \gamma_{x}$

$x \vDash \diamond \varepsilon_{y} \wedge \square\left(\nabla^{+} A t(x) . \rightarrow . \diamond \varepsilon_{y}\right)$ for all $\varepsilon_{y} \in S U C^{+}(x)$ by the fact that $x$ is $\operatorname{At}(x)$-maximal and $x \vDash \checkmark \varepsilon_{y}$ for all $\varepsilon_{y} \in S U C^{+}(x)$. Furthermore, $x \vDash \neg \vee \varepsilon_{y}$ for all $y \notin s u c^{+}(s)$ and $d^{\mu}(y) \leq d$. Finally, suppose for $x \triangleleft z$ that $z \vDash \neg \backslash \varepsilon_{y}$ for some $y \in \operatorname{suc}^{+}(x)$. By definition of depth, $d^{\mu}\left(z^{\mu}\right) \leq d$. Hence, by induction hypothesis $z^{\mu} \vDash \lambda_{x}$. If $z=z^{\mu}$ then $z \vDash \lambda_{x} \wedge \alpha$, if $z \triangleleft z^{\mu}$ then $z \vDash \diamond\left(\alpha \wedge \lambda_{x}\right)$ for $\alpha=\operatorname{At}(z) \in A$. And so

$$
z \vDash \zeta:=\bigvee\left\langle\diamond^{+}\left(\alpha \wedge \lambda_{x}\right): \alpha \in A\right\rangle
$$

from which $x=\square\left(\square \neg \varepsilon_{y} \rightarrow \zeta\right)$. This shows (iii). We have shown that $x=\varepsilon_{x}$.

(iv) Now suppose $y \vDash \lambda_{d+1}$. If also $y \vDash \lambda_{d}$ then $d^{\mu}(y) \leq d$, by induction hypothesis. Hence let $y \vDash \neg \lambda_{d}$. Then $y \vDash \varepsilon_{z}$ for some maximal $z$ with $d^{\mu}(z)=d+1$ and so $y \vDash \backslash \varepsilon_{x}$ for some maximal $x$ with $d^{\mu}(x)=d$. So, $d^{\mu}(y)>d$. But $y \vDash \beta_{z}$, implying that if $y \triangleleft v \vDash \square^{+} \neg A t(y)$, then $\operatorname{At}(v) \notin C L(z)$ and so $v \vDash \diamond^{+}\left(\operatorname{At}(v) \wedge \lambda_{d}\right)$. If $v \vDash \lambda_{d}, d^{\mu}(v) \leq d$; but if $v \vDash \diamond\left(A t(v) \wedge \lambda_{d}\right)$ then $v^{\mu} \vDash \lambda_{d}$ and so $d^{\mu}(v)=d^{\mu}\left(v^{\mu}\right) \leq d$ as well. This proves $d^{\mu}(y)=d+1$. 
Lemma 57 For all $x, y \in F$ :
(a) $\varepsilon_{x} \equiv \varepsilon_{y} \quad \Leftrightarrow \quad \vdash_{\mathbf{K} 4} \varepsilon_{x} \leftrightarrow \varepsilon_{y}$
(b) $\varepsilon_{x} \not \equiv \varepsilon_{y} \quad \Leftrightarrow \quad \vdash_{\mathbf{K} 4} \varepsilon_{x} \rightarrow \neg \varepsilon_{y}$
(c) $\varepsilon_{x} \triangleleft \varepsilon_{y} \quad \Leftrightarrow \quad \vdash_{\mathbf{K} 4} \varepsilon_{x} \rightarrow \diamond \varepsilon_{y}$
(d) $\varepsilon_{x} \not \varepsilon_{y} \quad \Leftrightarrow \quad \vdash_{\mathbf{K} 4} \varepsilon_{x} \rightarrow \neg \vee \varepsilon_{y}$

Proof. It is enough to show only $(\Rightarrow)$ in each case. (a) holds by definition of $\equiv$ and $\varepsilon_{x}$.

(b) If $S U C^{+}(x) \neq S U C^{+}(y)$, for example $\varepsilon_{w} \in S U C^{+}(x)-S U C^{+}(y)$, then $\vdash_{\mathbf{K} 4}$ $\varepsilon_{x} \rightarrow \diamond \varepsilon_{w} ; \varepsilon_{y} \rightarrow \neg \backslash \varepsilon_{w}$, whence $\vdash_{\mathbf{K} 4} \varepsilon_{x} \rightarrow \neg \varepsilon_{y}$; likewise for $\varepsilon_{w} \in S U C^{+}(y)-$ $S U C^{+}(x)$. Let us now suppose $S U C^{+}(x)=S U C^{+}(y)$. If $\operatorname{At}(x) \neq A t(y)$, the case is clear. Thus if $A t(x)=A t(y)$, we must have $C L(x) \neq C L(y)$. Without loss of generality we can assume that $\alpha \in C L(x)-C L(y)$. We have $\vdash_{\mathbf{K} 4} \varepsilon_{x} \rightarrow \diamond \alpha ; \varepsilon_{x} \rightarrow$ $\square\left(\diamond^{+} A t(x) \rightarrow \neg \lambda_{x}\right)$ (by $\vdash_{\mathbf{K} 4} \alpha_{x} \rightarrow \square\left(\diamond^{+} A t(x) . \rightarrow . \neg \lambda_{x}\right)$ and $\left.\vdash_{\mathbf{K} 4} \varepsilon_{x} \rightarrow \alpha_{x}\right)$. Furthermore, from $\vdash_{\mathbf{K} 4} \varepsilon_{x} \rightarrow \square(\alpha \rightarrow \diamond A t(x))$ we get $\vdash_{\mathbf{K} 4} \varepsilon_{x} \rightarrow \diamond\left(\alpha \wedge \neg \lambda_{x}\right)$. Also, $\varepsilon_{x} \vdash_{\mathbf{K} 4} \square(\alpha \rightarrow \diamond A t(x))$ (this is a conjunct of $\beta_{x}$ ), and $\varepsilon \vdash_{\mathbf{K} 4} \square\left(\diamond A t(x) \rightarrow \neg \lambda_{x}\right)$ (this follows from the last conjunct of $\left.\alpha_{x}\right)$. Together this gives $\varepsilon_{x} \vdash_{\mathbf{K} 4} \square\left(\alpha \rightarrow \neg \lambda_{x}\right)$. But

$$
\vdash_{\mathbf{K} 4} \varepsilon_{y} \rightarrow \square\left(\alpha \rightarrow \diamond^{+}\left(\alpha \wedge \lambda_{y}\right)\right) .
$$

By definition of $\beta_{y}$ and since $S U C^{+}(x)=S U C^{+}(y)$ we have $\lambda_{x} \equiv \lambda_{y}$. Consequently $\vdash_{\mathbf{K} 4} \varepsilon_{y} \rightarrow \square\left(\alpha \rightarrow \diamond^{+}\left(\alpha \wedge \lambda_{x}\right)\right)$. Now

$$
\varepsilon_{x} \wedge \varepsilon_{y} \vdash_{\mathbf{K} 4} \diamond\left(\alpha \wedge \neg \lambda_{x}\right) ; \square\left(\alpha \rightarrow \neg \lambda_{x}\right) ; \square\left(\alpha \rightarrow \diamond^{+}\left(\alpha \wedge \lambda_{x}\right)\right) \vdash_{\mathbf{K} 4} \perp .
$$

(The last two formulae give $\square\left(\alpha \rightarrow \diamond^{+}\left(\alpha \wedge \lambda_{x} \wedge \neg \lambda_{x}\right)\right)$, by transitivity; therefore, $\square\left(\alpha \rightarrow \square^{+} \perp\right)$ is derivable, which gives $\square \neg \alpha$. $\diamond \alpha$ is derivable from the first formula. Hence, $\perp$ is derivable.) So, $\vdash_{\mathbf{K} 4} \varepsilon_{x} \rightarrow \neg \varepsilon_{y}$.

(c) If $\varepsilon_{y} \in S U C^{+}(x)$, the case is trivial. So let us suppose the contrary. Then $x \triangleleft y \triangleleft x$ and so $\operatorname{cl}(x)=\operatorname{cl}(y)$ as well as $\operatorname{suc}^{+}(x)=\operatorname{suc}^{+}(y)$. Hence $\operatorname{SUC}^{+}(x)=$ $S U C^{+}(y)$ and $C L(x)=C L(y) \neq \emptyset$. Since $\varepsilon_{y}$ is a conjunction of formulae $\psi_{i}, i<n$, in order to show $\vdash_{\mathbf{K} 4} \varepsilon_{x} \rightarrow \diamond \varepsilon_{y}$ it is enough to show $\vdash_{\mathbf{K} 4} \varepsilon_{x} \rightarrow \diamond \psi_{0}$ and $\vdash_{\mathbf{K} 4} \varepsilon_{x} \rightarrow$ $\square\left(\psi_{0} \rightarrow \psi_{i}\right), 0<i<n$. We take $\psi_{0}:=\operatorname{At}(y)$. Furthermore, $\beta_{x}=\beta_{y}$ and $\gamma_{x}=\gamma_{y}$, from which it easily follows with the help of $\vdash_{\mathbf{K} 4} \varepsilon_{x} \rightarrow \square\left(\operatorname{At}(y) \rightarrow \diamond^{+} \operatorname{At}(x)\right)$ that $\vdash_{\mathbf{K} 4} \varepsilon_{x} \rightarrow \square\left(A t(y) . \rightarrow \beta_{y} \wedge \gamma_{y}\right)$. Now

(i) $\vdash_{\mathbf{K} 4} \varepsilon_{x} \rightarrow \diamond A t(y)$, since $\operatorname{At}(y) \in C L(x)$.

(ii) $\vdash_{\mathbf{K} 4} \varepsilon_{x} \rightarrow \square\left(A t(y) \rightarrow \neg \lambda_{y}\right)$ follows from $\vdash_{\mathbf{K} 4} \varepsilon_{x} \rightarrow \square(\operatorname{At}(y) \rightarrow \diamond A t(x))$, $\vdash_{\mathbf{K} 4} \varepsilon_{x} \rightarrow \square\left(\diamond A t(x) \rightarrow \neg \lambda_{x}\right)$ and $\lambda_{x} \equiv \lambda_{y}$. 
(iii) $\vdash_{\mathbf{K} 4} \varepsilon_{x} \rightarrow \square\left(\operatorname{At}(y) \rightarrow \square\left(\lambda_{y} \rightarrow \neg \nabla^{+} A t(y)\right)\right)$. For $\vdash_{\mathbf{K} 4} \varepsilon_{x} \rightarrow \square\left(\lambda_{x} \rightarrow\right.$ $\left.\neg \diamond^{+} A t(x)\right)$, whence $\vdash_{\mathbf{K} 4} \varepsilon_{x} \rightarrow \square\left(\lambda_{y} \rightarrow \diamond^{+} A t(y)\right)$. For $\lambda_{x}=\lambda_{y}$, and furthermore $\vdash_{\mathbf{K} 4} \varepsilon_{x} \rightarrow \square\left(A t(y) \rightarrow \diamond^{+} A t(x)\right)$. The claim now easily follows.

(i), (ii) and (iii) together give $\vdash_{\mathbf{K} 4} \varepsilon_{x} \rightarrow \diamond \alpha_{y}$. Thus $\vdash_{\mathbf{K} 4} \varepsilon_{x} \rightarrow \diamond \varepsilon_{y}$, as required.

(d) Case 1. If $d^{\mu}(y)<d^{\mu}(x)$, then $\neg \backslash \varepsilon_{y}$ is a conjunct of $\varepsilon_{x}$.

Case 2. $d^{\mu}(x)=d^{\mu}(y)$. Then $\lambda_{x} \equiv \lambda_{y}$. Suppose $\varepsilon_{u} \in S U C^{+}(x)-S U C^{+}(y)$ for some $\varepsilon_{u}$. If $\vdash_{\mathbf{K} 4} \varepsilon_{x} \rightarrow \diamond \varepsilon_{y}$ then we have

$$
\vdash_{\mathbf{K} 4} \varepsilon_{y} . \rightarrow . \neg \vee \varepsilon_{u} \wedge \neg \lambda_{x} \wedge \bigwedge\left\langle\alpha \rightarrow \neg \nabla^{+}\left(\alpha \wedge \lambda_{x}\right): \alpha \in A(\varphi)-C L(x)\right\rangle
$$

So we get

$$
\vdash_{\mathbf{K} 4} \varepsilon_{x} \rightarrow \diamond\left(\square \neg \varepsilon_{u} . \wedge \neg \lambda_{x} \wedge \bigwedge\left\langle\alpha \rightarrow \diamond^{+}\left(\alpha \wedge \lambda_{x}\right): \alpha \in A(\varphi)-C L(x)\right\rangle\right) .
$$

But this is a contradiction to $\vdash_{\mathbf{K} 4} \varepsilon_{x} \rightarrow \gamma_{x}$. Now suppose $\varepsilon_{u} \in S U C^{+}(y)-S U C^{+}(x)$. Then $\vdash_{\mathbf{K} 4} \varepsilon_{x} \rightarrow \diamond \varepsilon_{y}$ yields $\vdash_{\mathbf{K} 4} \varepsilon_{x} \rightarrow \diamond \varepsilon_{u}$ in contradiction to $\vdash_{\mathbf{K} 4} \varepsilon_{x} \rightarrow \square \neg \varepsilon_{u}$. Thus the case $S U C^{+}(x)=S U C^{+}(y)$ is left. Then we must have $C L(x) \neq C L(y)$ or $C L(x)=C L(y)=\emptyset$. The latter case is dealt with as follows. $\vdash_{\mathbf{K} 4} \varepsilon_{x} \rightarrow \diamond \varepsilon_{y}$ implies $\vdash_{\mathbf{K} 4} \varepsilon_{x} \rightarrow \diamond A t(y)$; and since $\varepsilon_{x} \vdash_{\mathbf{K} 4} \square\left(A t(y) \rightarrow \diamond^{+}\left(\operatorname{At}(y) \wedge \lambda_{y}\right)\right.$ ) (for $\operatorname{At}(y) \notin C L(x))$ we have $\vdash_{\mathbf{K} 4} \varepsilon_{x} \rightarrow \diamond\left(\varepsilon_{y} \wedge \diamond^{+}\left(\operatorname{At}(y) \wedge \lambda_{y}\right)\right)$, in contradiction to $\varepsilon_{y} \vdash_{\mathbf{K} 4} \neg \lambda_{y} \wedge \square\left(\lambda_{y} \rightarrow \neg A t(y)\right)$. Thus $C L(x) \neq C L(y)$. Now let $\alpha \in C L(y)-C L(x)$. Then $\vdash_{\mathbf{K} 4} \varepsilon_{x} \rightarrow \square\left(\alpha \rightarrow \diamond^{+}\left(\alpha \wedge \lambda_{x}\right)\right)$ and $\vdash_{\mathbf{K} 4} \varepsilon_{y} \rightarrow \square\left(\alpha \rightarrow \neg \lambda_{x}\right)$ and if $\vdash_{\mathbf{K} 4}$ $\varepsilon_{x} \rightarrow \diamond \varepsilon_{y}$ we get $\vdash_{\mathbf{K} 4} \varepsilon_{x} \rightarrow \diamond\left(\alpha \wedge \lambda_{x}\right) \wedge \square\left(\alpha \rightarrow \neg \lambda_{x}\right)$, again a contradiction. Assume finally $\gamma \in C L(x)-C L(y)$. Then $\vdash_{\mathbf{K} 4} \varepsilon_{x} \rightarrow \square(A t(y) \rightarrow \diamond \gamma)$ and $\vdash_{\mathbf{K} 4} \varepsilon_{y} \rightarrow$ $\square\left(\gamma \rightarrow \diamond^{+}\left(\gamma \wedge \lambda_{x}\right)\right)$. We arrive at a contradiction with $\vdash_{\mathbf{K} 4} \varepsilon_{x} \rightarrow \nabla_{y}$ because $\vdash_{\mathbf{K} 4} \varepsilon_{x} \rightarrow \square\left(\gamma \rightarrow \neg \lambda_{x}\right)$.

Case 3. $d^{\mu}(y)>d^{\mu}(x)$. If there is a $z$ with $d^{\mu}(z)=d^{\mu}(x), \varepsilon_{y} \triangleleft \varepsilon_{z}$ and $\varepsilon_{z} \not \varepsilon_{x} \not$ $\varepsilon_{z}$, then $\vdash_{\mathbf{K} 4} \varepsilon_{x} \rightarrow \diamond \varepsilon_{y}$ would imply $\vdash_{\mathbf{K} 4} \varepsilon_{x} \rightarrow \diamond \varepsilon_{z}$, which is contradiction because of Case 2. But in the other case $\varepsilon_{y} \triangleleft \varepsilon_{x}$ and since $\vdash_{\mathbf{K} 4} \varepsilon_{y} \rightarrow \square\left(\lambda_{y} \rightarrow \square \neg A t(y)\right)$ and $\vdash_{\mathbf{K} 4} \varepsilon_{x} \rightarrow \lambda_{y}$ we get $\vdash_{\mathbf{K} 4} \varepsilon_{y} \rightarrow \square\left(\varepsilon_{x} \rightarrow \square \neg A t(y)\right)$ showing $\vdash_{\mathbf{K} 4} \varepsilon_{x} \rightarrow \neg \vee \varepsilon_{y}$.

Since the depth of a point in $\Re$ is bounded we have a bounded number of formulae $\varepsilon_{x}$ no matter what the frame $\mathfrak{F}$ is we started with. This number we denote by $c(\varphi)$. It can be computed from $\varphi$, but we will not do that here. Another consequence of Lemma 57 is

Proposition 58 Let $\mathfrak{G}$ be a finite frame and $\mathfrak{F}^{\mu}$ be subreducible to $\mathfrak{G}$, that is, $\mathfrak{F}^{\mu} \supseteq \mathfrak{H} \stackrel{\pi}{\rightarrow} \mathfrak{G}$, and let $\chi_{y}:=\bigvee\left\langle\varepsilon_{w}: w \in h, \pi(w)=y\right\rangle$, for $y \in G$. Then

$$
\vdash_{\mathbf{K} 4} \operatorname{SF}(\mathfrak{G})\left[\chi_{y} / p_{y}\right]
$$


where $\varphi\left[\chi_{y} / p_{y}\right]$ is the result of replacing $\chi_{y}$ for all occurrences of $p_{y}$ for all $y$ in $\varphi$.

Proof. It is easily seen that $\vdash_{\mathbf{K} 4} \chi_{v} \rightarrow \neg \chi_{w}$ if $v \neq w, \vdash_{\mathbf{K} 4} \chi_{v} \rightarrow \diamond \chi_{w}$ if $v \triangleleft w$ and $\vdash_{\mathbf{K} 4} \chi_{v} \rightarrow \neg \vee \chi_{w}$ if $v \Varangle w$.

Proposition 59 The map $\rho: \mathfrak{F}^{\mu} \rightarrow \mathfrak{R}$ given by $\rho: x \mapsto \varepsilon_{x}$ is a p-morphism admissible for $\beta$. Moreover, let $\mathbb{D}$ be the algebra of sets generated by the sets $\rho[\beta(p)]=\left\{\alpha \in \delta: \alpha \vdash_{\mathbf{K} 4} p\right\}$. Then $\langle\mathfrak{d e s}, \mathbb{D}\rangle$ is refined.

Proof. Let $x \triangleleft y$. Then either $y \triangleleft x$ or $y \not x . \quad y \not x$ implies $\varepsilon_{x} \triangleleft \varepsilon_{y}$ by definition, since $\diamond \varepsilon_{y}$ is a conjunct of $\varepsilon_{x}$; if $y \triangleleft x$ then we have $S U C^{+}(x)=S U C^{+}(y)$, $C L(x)=C L(y)$ and $A t(y) \in C L(x)$. Thus $\varepsilon_{x} \triangleleft \varepsilon_{y}$ as in the proof for Lemma 57(c). Hence $x \triangleleft y$ implies $\varepsilon_{x} \triangleleft \varepsilon_{y}$. Furthermore, if $\rho(x) \triangleleft \varepsilon_{y}$ then since $\left\langle\mathfrak{F}^{\mu}, \beta, x\right\rangle \vDash$ $\varepsilon_{x}$ and $\vdash_{\mathbf{K} 4} \varepsilon_{x} \rightarrow \backslash \varepsilon_{y},\left\langle\mathfrak{F}^{\mu}, \beta, z\right\rangle \vDash \varepsilon_{y}$ for some $x \triangleleft z$. Then $\varepsilon_{y} \equiv \varepsilon_{z}$, that is, $\rho(z)=\varepsilon_{y}$. This shows that $\rho$ is a p-morphism. $\rho$ is clearly admissible; let $\gamma$ be the induced valuation on $\mathfrak{R}$. Because of $\left\langle\mathfrak{F}^{\mu}, \beta, z\right\rangle \vDash \varepsilon_{x}$, we have $\langle\mathfrak{R}, \gamma, \rho(z)\rangle \vDash \varepsilon_{x}$ but $\langle\mathfrak{R}, \gamma, \rho(z)\rangle \vDash \neg \varepsilon_{y}$ for $\varepsilon_{x} \neq \varepsilon_{y}$ and consequently $\langle\mathfrak{R}, \gamma\rangle$ is refined.

We have now constructed formulas $\varepsilon_{x}$ which completely describe the structure of the refined submodel of maximal points of any given finite model.

Theorem 60 Let $L \supseteq K_{4}$ be a subframe logic, $\mathfrak{F}$ a finite, rooted frame. If $L$ has the finite model property, $L /_{F} \mathfrak{F}$ has the finite model property as well. Moreover, if $\varphi$ is consistent with $L /_{F} \mathfrak{F}$ then it has a model of size $\leq c(\varphi)$.

Proof. By constructive reduction. Suppose $\varphi$ is consistent with $L / \mathrm{F} \mathfrak{F}$. Then define $\varphi^{\sharp}$ to be the union of the sets $F(\langle\Re, \beta\rangle)$ where $\mathfrak{R}$ is of cardinality $\leq c(\varphi), \beta$ a valuation from $\operatorname{var}(\varphi)$ into $\Re$ and

$$
F(\langle\Re, \beta\rangle):=\left\{\square^{+} C_{g}\left[\varepsilon_{S(x)} / p_{x}\right]: S: G \rightarrow 2^{r}\right\}, \varepsilon_{S(x)}:=\bigvee\left\langle\varepsilon_{w}: w \in S(x)\right\rangle
$$

So $\varphi ; \varphi^{\sharp}$ is $L_{\mathfrak{F}}$-consistent and a fortiori $L$-consistent. Therefore $\langle\mathfrak{Z}, \zeta, w\rangle \vDash \varphi ; \varphi^{\sharp}$ for some $L$-model $\langle\mathfrak{Z}, \zeta, w\rangle$. Let $\mathfrak{R}$ be the reduced subframe of maximal points. We have $\left\langle\mathfrak{R}, \zeta, \varepsilon_{w}\right\rangle \vDash \varphi$, by Proposition 59. Now suppose $\mathfrak{R} \supseteq \mathfrak{H} \stackrel{\pi}{\rightarrow} \mathfrak{F}$. Then let $S: y \mapsto \pi^{-1}(y)$. If $v \in \pi^{-1}(x)$ with $x$ generating $\mathfrak{F}$ then $\left\langle\mathfrak{Z}^{\mu}, \zeta, v\right\rangle \vDash \neg C_{g}\left[\varepsilon_{S(y)} / p_{y}\right]$ since $\left\langle\mathfrak{Z}^{\mu}, \zeta, v\right\rangle \vDash \varepsilon_{S(s)}$ and $\left\langle\mathfrak{Z}^{\mu}, \zeta\right\rangle \vDash S F(\mathfrak{F})\left[\varepsilon_{S(x)} / p_{x}\right]$, by Proposition 58. Thus $v \notin z$. Consequently, $\mathfrak{R}$ is not subreducible to $\mathfrak{F}$. $\langle\mathfrak{R}, \zeta, w\rangle$ is an $L_{\mathfrak{F}}-$ model for $\varphi$ and $\operatorname{card}\left(\Re_{w}\right) \leq c(\varphi)$. 
Analogously the same theorem for cofinal subframe logics is proved. By induction one can now show that all Fine-splittings K4/F $G$ (and all Zakharyaschevsplittings $\mathbf{K} / \mathrm{z} H$ for finite $H$ ) have the finite model property. But this is all we need to show the full theorem. Let $\varphi$ be $\mathbf{K 4} /{ }_{\mathrm{F}} G$-consistent. Define $G^{\varphi}:=\{\mathfrak{G} \in G$ : $\operatorname{card}(\mathfrak{G}) \leq c(\varphi)\}$. Then $\varphi$ is $\mathbf{K 4} /{ }_{\mathrm{F}} G^{\varphi}$-consistent and has a finite model of size $\leq c(\varphi)$ by the preceding theorem. But this already is a $\mathbf{K 4} /{ }_{\mathrm{F}} G-$ model. This completes the proof of Theorem 53

\section{References}

[1] Philippe Balbiani and Andreas Herzig. A translation from the modal logic of provability into K4. Journal of Applied Non-Classical Logics, 4(1):73-78, 1994.

[2] Alexander Chagrov and Michael Zakharyaschev. Modal Logic. Oxford University Press, Oxford, 1997.

[3] Kit Fine. Logics containing K4, Part II. Journal of Symbolic Logic, 50:619$651,1985$.

[4] Dov M. Gabbay and Valentin B. Shehtman. Undecidability of modal and intermediate logics with two individual variables. Journal of Symbolic Logic, 58:800 - 823, 1993.

[5] Giuseppe de Giacomo. Eliminating "Converse" from Converse PDL. Journal of Logic, Language and Information, 5:193-208, 1996.

[6] Valentin Goranko and Solomon Passy. Using the universal modality: Gains and Questions. Journal of Logic and Computation, 2:5-30, 1992.

[7] Marcus Kracht. Tools and Techniques in Modal Logic. Number 142 in Studies in Logic. Elsevier, Amsterdam, 1999.

[8] Marcus Kracht and Frank Wolter. Properties of independently axiomatizable bimodal logics. Journal of Symbolic Logic, 56:1469-1485, 1991.

[9] Ekkehard Krätzel. Zahlentheorie. VEB Deutscher Verlag der Wissenschaften, Berlin, 1981. 
[10] Robert E. Ladner. The computational complexity of provability in systems of modal logic. SIAM Journal of Computing, 6:467 - 480, 1977.

[11] Larisa L. Maksimova. The Beth Properties, interpolation, and amalgamability in varieties of modal algebras. Soviet Mathematical Doklady, 44:327$331,1992$.

[12] Larisa L. Maksimova. Definability and interpolation in classical modal logics. Contemporary Mathematics, 131:583-599, 1992.

[13] Larisa L. Maksimova. On variable separation in modal and superintuitionistic logics. Studia Logica, 55:99-112, 1995.

[14] Wolfgang Rautenberg. Modal tableau calculi and interpolation. Journal of Philosophical Logic, 12:403-423, 1983.

[15] Edith Spaan. Complexity of Modal Logics. PhD thesis, Department of Mathematics and Computer Science, University of Amsterdam, 1993.

[16] Michael Zakharyaschev. Canonical formulas for K4, Part II: Cofinal Subframe Logics. Journal of Symbolic Logic, 61:421-449, 1996. 